JOURNAL OF

SYMPLECTIC GEOMETRY

Volume 11, Number 2, 231-268, 2013

\title{
DISPLACEMENT OF POLYDISKS AND LAGRANGIAN FLOER THEORY
}

Kenji Fukaya, Yong-Geun Oh, Hiroshi Ohta, and Kaoru Ono

There are two purposes of the present paper. One is to correct an error in the proof of Theorem 6.1.25 in [3], from which Theorem $\mathrm{J}[\mathbf{3}]$ follows. In the course of doing so, we also obtain a new lower bound of the displacement energy of polydisks in general dimension.

\section{Contents}

1. Introduction

2. Notations

3. A non-displacement theorem

4. Comparison of two Cauchy-Riemann equations and intertwining transformation

5. Improved energy estimate

6. Corrected proofs of Theorem $\mathbf{J}$ and Theorem 6.1.25 [3] 21

6.1. Statement of Theorem $\mathbf{J}[3]$

6.2. Proof of Theorem 6.2

7. Torsion threshold and displacement energy 28

7.1. Torsion threshold 28

7.2. $\mathfrak{P O}$-threshold for toric fibers $\quad 29$

8. Displacement of polydisks inside cylinders in high dimensions

9. Appendix. 


\section{Introduction}

In [3], we stated a lower bound of the displacement energy of relatively spin Lagrangian submanifold $L$ with a bounding cochain $b$ in terms of torsion exponents of Floer cohomology $H F\left((L, b),(L, b) ; \Lambda_{0, \text { nov }}\right)$. This is Theorem $\mathrm{J}$ in [3], which is a consequence of Theorem 6.1.25 in [3]. See Theorems 6.1 and 6.2 of this paper for the precise statement in a general setting. However, the proof of Theorem 6.1.25 contains an inaccurate point. One of the purposes of the present paper is to correct this error. There are two key ingredients in this correction of the error, which are interrelated to each other: one is the usage of an optimal Floer chain map introduced in the present paper, and an energy estimate of the type which was first introduced by Chekanov [1] and clarified by the second named author in [12].

Another purpose is to employ Theorem $\mathrm{J}[\mathbf{3}]$ and obtain an estimate of the lower bound of the displacement energy of polydisks in cylinder.

Before we achieve the two purposes above, we prove the following nondisplacement theorem to illustrate a geometric consequence of Theorem $\mathrm{J}[\mathbf{3}]$ in a simple example. Let $S^{1}(S) \subset \mathbb{C}$ be a circle of radius $\sqrt{\pi^{-1} S}$. We also consider $S_{\text {eq }}^{1} \subset S^{2}(1)$ the equator in the sphere of area 1.

Theorem 1.1. We put $X=\mathbb{C} \times S^{2}(1)$. Consider any time-dependent Hamiltonian $H:[0,1] \times X \rightarrow \mathbb{R}$ with its Hofer norm $\|H\|<S$. Then we have

$$
\psi_{H}\left(S^{1}(S) \times S_{e q}^{1}\right) \cap\left(S^{1}(S) \times S_{e q}^{1}\right) \neq \emptyset
$$

for its time-one map $\psi_{H}:=\phi_{H}^{1}$.

When $S \leq 1 / 2$, the above statement is a direct consequence of Chekanov's theorem [1]. Thus the new result is the case that $S>1 / 2$. The proof of this theorem is 'elementary' in that it uses only the Lagrangian Floer theory for monotone Lagrangian submanifolds $[\mathbf{1 0}]$ and by now standard computations for the energy estimates used as in [1], [12], but does not use any techniques of virtual fundamental chains, Bott-Morse theory or any higher homological algebra.

In fact, this theorem is a corollary of Theorem $\mathrm{J}[\mathbf{3}]$ whose precise statement we refer to Section 6 . We provide this elementary proof in this particular case partly because it nicely illustrates Theorem $\mathrm{J}[\mathbf{3}]$ when a deformation of Floer cohomology by a bounding cochain is not needed. (On the other hand, the proof of Theorem $\mathrm{J}[\mathbf{3}]$ is given in a very general context in terms of the deformed Floer cohomology of weakly unobstructed Lagrangian submanifolds after bulk deformations.)

Remark 1.2. Let $e^{Z_{1,1}}(D(1, S))$ be the displacement energy (Definition 2.1 $(2))$ of the bidisk $D(1, S)$ in the cylinder $Z_{1,1}=Z_{1,1}(1+\varepsilon)$. Here we denote the polydisks in $\mathbb{C}^{2}$ by

$$
D(a, b)=\left\{\left.\left(z_{1}, z_{2}\right)|\pi| z_{1}\right|^{2}<a, \pi\left|z_{2}\right|^{2}<b\right\}=D^{2}(a) \times D^{2}(b) \subset \mathbb{C}^{2}
$$


with $a \leq b$, and also denote the cylinder in $\mathbb{C}^{2}$ by

$$
Z_{1,1}(a+\varepsilon)=\left\{\left.\left(z_{1}, z_{2}\right)|\pi| z_{1}\right|^{2}<a+\varepsilon\right\}=D^{2}(a+\varepsilon) \times \mathbb{C}
$$

for $\varepsilon>0$ small. Theorem $\mathrm{J}[\mathbf{3}]$ implies the following lower bound

$$
S \leq e^{Z_{1,1}}(D(1, S))
$$

whenever $S>1$. We could find a certain claim related to $e^{Z_{1,1}}(D(1, S))$ in literatures in the past. The lower bound (1.1) is optimal.

Our proof relies on the study of torsion thresholds of Floer cohomology of Lagrangian torus fiber and Theorem J [3].

However, although the statement of Theorem J [3] is correct as it is, its proof contains some incorrect argument at the end of the proof of Theorem 6.1.25 in p. 392: The homomorphisms in line 9 and 11 of page 392 of [3] is not well-defined. See also Remark 4.5. Moreover, to give a correct proof of the same statement as stated in Theorem 6.1.25, hence Theorem J, we also need to improve the energy estimate given in Proposition 5.3.45 [3] and use a different construction of a Floer chain map. It turns out that to obtain the optimal energy estimate needed to prove Theorem $\mathrm{J}$ in the construction of a Floer chain map, we need to use the Hamiltonian perturbed Cauchy-Riemann equation with fixed Lagrangian boundary condition which intertwines the so-called geometric version of the Floer cohomology and the dynamical version of the Floer cohomology and then applying the intertwining transformation that relates the two. Similar transformation was used by the second named author previously in $[\mathbf{1 1}]$ for a similar purpose. Using this trick and an optimal energy estimate originated by Chekanov [1] , we can prove the statement of Theorem 6.1.25 and hence Theorem $\mathrm{J}$ in $[\mathbf{3}]$ as they are currently stated.

Another purpose of the present paper is to apply Theorem $\mathrm{J}[\mathbf{3}]$ in the study of displacement energy of polydisks in arbitrary dimension and generalize the above mentioned four-dimensional result to arbitrary dimension whose description is now in order. It turns out that the Lagrangian Floer theory developed in $[\mathbf{3}]$ and $[\mathbf{4}, \boldsymbol{5}]$ can be nicely applied to the various symplectic topological questions concerning polydisks $D^{2}\left(r_{1}\right) \times \cdots \times D^{2}\left(r_{n}\right)$. This is largely because the polydisks contain Lagrangian tori which can be embedded into the toric manifolds $S^{2}\left(a_{1}\right) \times \cdots \times S^{2}\left(a_{n}\right)$ or $S^{2}(a) \times \mathbb{C} P^{n-1}(\lambda)$ for suitable choices of $a_{i}$ 's or $(a, \lambda)$. Here $\mathbb{C} P^{n-1}(\lambda)$ is the projective space with the Fubini-Study Kähler form $\omega$ with $[\omega](C)=\lambda$ for the homology class $C$ of the complex line. With this notation, we have the symplectic embedding of $B^{2 n-2}(\lambda) \hookrightarrow \mathbb{C} P^{n-1}(\lambda)$ such that $\mathbb{C} P^{n-1}(\lambda) \backslash B^{2 n-2}(\lambda)$ is the hyperplane at infinity. In this way, we obtain various improvements and generalizations of the theorems concerning symplectic topology of polydisks proven in $[\mathbf{8}]$. See [7] for other applications of Lagrangian Floer theory a la [3-5]. 
We give two high-dimensional generalizations of $(1.1)$. Let $\left(z_{1}, \ldots, z_{n}\right)$ be the complex coordinates of $\mathbb{C}^{n} \cong \mathbb{R}^{2 n}$. We decompose

$$
\left(z_{1}, \ldots, z_{n}\right)=\left(z_{1}, z^{\prime}\right)
$$

with $z^{\prime}=\left(z_{2}, \ldots, z_{n}\right)$. We denote

$$
D\left(a_{1}, a_{2}, \ldots, a_{n}\right)=\left\{\left.\left(z_{1}, \ldots, z_{n}\right)|\pi| z_{1}\right|^{2}<a_{1}, \ldots, \pi\left|z_{n}\right|^{2}<a_{n}\right\} \subset \mathbb{C}^{n}
$$

where $a_{1} \leq a_{2} \leq \cdots \leq a_{n}$. We also denote the cylinder over the disk $\left|z_{1}\right|^{2} \leq\left(a_{1}+\varepsilon\right) / \pi$ by

$$
Z_{1, n-1}\left(a_{1}+\varepsilon\right)=\left\{\left.\left(z_{1}, \ldots, z_{n}\right)|\pi| z_{1}\right|^{2}<a_{1}+\varepsilon\right\}
$$

for $\varepsilon>0$ small. Then we have the following two theorems.

Theorem 1.3. Let $S>1$ and $0<\varepsilon<1$. Put $Z_{1, n-1}=Z_{1, n-1}(1+\varepsilon)$. Then we have

$$
S \leq e^{Z_{1, n-1}}(D(1, S, \ldots, S)) .
$$

Theorem 1.4. Let $S>1$ and $0<\varepsilon<1$. Let $k$ be an integer satisfying $1 \leq k<n$. Put $Z_{n-k, k}=Z_{n-k, k}(1+\varepsilon)=D^{2}(1+\varepsilon)^{n-k} \times \mathbb{C}^{k}$. Then we have

$$
S \leq e^{Z_{n-k, k}}\left(D^{2}(1)^{n-k} \times B^{2 k}(k S)\right) .
$$

This paper borrows many notations and definitions from [3] without delving into detailed explanations thereof. Especially, the notion of bulk deformations is used mainly to make the statement of Theorem $\mathrm{J}$ from $[\mathbf{3}]$ in this paper as close as possible to that of $[\mathbf{3}]$. We refer to the relevant sections of $[\mathbf{3}]$ for more explanations thereof. However, for those who are mainly interested in the overall argument how the torsion threshold can be used in the study of displacement energy, we recommend them to directly look at Section 3, Section 7 and Section 8 and refer to other sections as needed.

In March 2010, R. Hind gave a talk at MSRI workshop on Symplectic and Contact Topology and Dynamics: Puzzles and Horizons. Thanks to his talk, we took another look at our proof of Theorem 6.1.25 in [3] and found out an inaccurate point in the proof, which we rectify in this paper. We thank him for his interesting talk and discussion.

\section{Notations}

We introduce the following general definitions and notations that we will use in this paper.

Let $(X, \omega)$ be a symplectic manifold. We denote by $J$ a time-dependent family of $\omega$-compatible almost complex structures $J=\left\{J_{t}\right\}_{t \in[0,1]}$. 
Let $\psi: X \rightarrow X$ be a Hamiltonian diffeomorphism and $H \in C^{\infty}([0,1] \times X)$ a normalized Hamiltonian with $\phi_{H}^{1}=\psi$ and $\int_{X} H_{t} \omega^{n}=0$. We denote the Hofer norm (see [9]) of $H$ by

$$
\|H\|=\int_{0}^{1}\left(\max H_{t}-\min H_{t}\right) d t .
$$

We define the Hofer norm of $\psi$ by

$$
\|\psi\|=\inf _{H \mapsto \psi}\|H\|,
$$

where $H \mapsto \psi$ means that $\psi=\phi_{H}^{1}$. We also define the length of a Hamiltonian isotopy $\phi_{H}=\left\{\phi_{H}^{t}\right\}$ by

$$
\operatorname{leng}\left(\phi_{H}\right)=\|H\| \text {. }
$$

Following Weinstein's notation [13], we denote the set of Hamiltonian deformations of $L$ by

$$
\mathfrak{I s o}(L)=\{\psi(L) \mid \psi \in \operatorname{Ham}(X, \omega)\}
$$

for a given Lagrangian submanifold $L \subset(X, \omega)$.

Definition 2.1. (1) Let $L^{\prime} \in \mathfrak{I s o}(L)$. Then we define the Hofer distance between $L, L^{\prime}$ by

$$
\operatorname{dist}\left(L, L^{\prime}\right)=\inf _{H \in C^{\infty}([0,1] \times X)}\left\{\|H\| \mid \phi_{H}^{1}(L)=L^{\prime}\right\} .
$$

Or equivalently,

$$
\operatorname{dist}\left(L, L^{\prime}\right)=\inf _{\psi \in \operatorname{Ham}(X, \omega)}\left\{\|\psi\| \mid \psi(L)=L^{\prime}\right\} .
$$

(2) Let $Y \subset X$. We define the displacement energy $e^{X}(Y) \in[0, \infty]$ by

$$
e^{X}(Y):=\inf _{\psi \in \operatorname{Ham}(X, \omega)}\{\|\psi\| \mid \psi(Y) \cap \bar{Y}=\emptyset\} .
$$

We put $e^{X}(Y)=\infty$ if there exists no $\psi \in \operatorname{Ham}(X, \omega)$ with $\psi(Y) \cap \bar{Y}=\emptyset$. When no confusion can occur, we simply write $e(Y)$ instead of $e^{X}(Y)$.

Let $\chi(\tau)$ be a smooth function on $\mathbb{R}$ such that $\chi(\tau)=0$ or 1 when $|\tau|$ is sufficiently large. In this paper, we will take $\chi(\tau)$ as one of the following three types, either

$$
\begin{aligned}
& \chi_{+}(\tau)= \begin{cases}0 & \text { for } \tau \leq 0, \\
1 & \text { for } \tau \geq 1,\end{cases} \\
& \chi_{+}^{\prime}(\tau) \geq 0,
\end{aligned}
$$


or $\chi_{-}=1-\chi_{+}$or $\chi_{K}$ satisfying

$$
\begin{aligned}
& \chi_{K}(\tau)= \begin{cases}0 & \text { for }|\tau| \geq K, \\
1 & \text { for }|\tau| \leq K-1,\end{cases} \\
& \chi_{K}^{\prime} \geq 0 \quad \text { on }[-K,-K+1], \quad \chi_{K}^{\prime} \leq 0 \quad \text { on }[K-1, K]
\end{aligned}
$$

for $K \geq 1$ and $\chi_{K}$ goes down to $\chi_{K=0}=0$, e.g., $\chi_{K}=K \chi_{1}$. (See (5.6).)
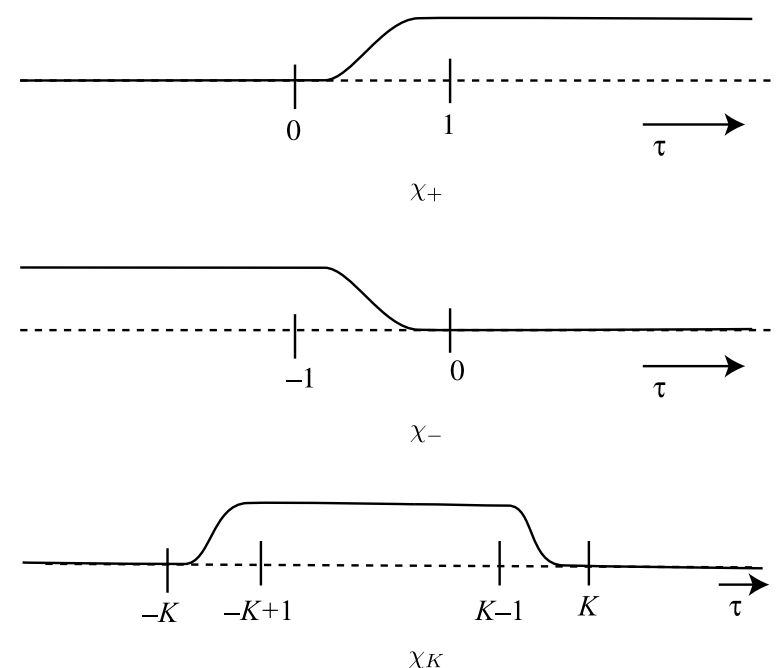

Ordering of the arguments in the pair $\left(L^{(0)}, L^{(1)}\right)$ varies in the notations from $[\mathbf{3}]$ for the various objects associated to the pair of Lagrangian submanifold $\left(L^{(0)}, L^{(1)}\right)$. We mostly follow them in the present paper. Specifically, we would like to mention the following conventions:

(1) (Path spaces) $\Omega\left(L^{(0)}, L^{(1)} ; \ell_{a}\right)$.

(2) (Floer moduli spaces) $\mathcal{M}\left(L^{(1)}, L^{(0)}\right)$.

(3) (Floer complex and homology) $C F\left(L^{(1)}, L^{(0)}\right), H F\left(L^{(1)}, L^{(0)}\right)$.

\section{A non-displacement theorem}

In this section, we prove Theorem 1.1. Recall that $S^{1}(S) \subset \mathbb{C}$ is a circle of radius $r$ with its area $\pi r^{2}=S$ and $S_{\text {eq }}^{1} \subset S^{2}(1)$ is the equator in the sphere of area 1 . We put $X=\mathbb{C} \times S^{2}(1)$.

Proof of Theorem 1.1. Suppose to the contrary that

$$
\psi_{H}\left(S^{1}(S) \times S_{\text {eq }}^{1}\right) \cap\left(S^{1}(S) \times S_{\text {eq }}^{1}\right)=\emptyset
$$

for a Hamiltonian $H$ with $\|H\|<S$. We denote

$$
c:=S-\|H\|>0 \text {. }
$$


Let $L^{(0)}=S^{1}(S) \times S_{\text {eq }}^{1}$. We choose $L^{(1)}$ as a small Hamiltonian perturbation of $S^{1}(S) \times S_{\text {eq }}^{1}$ defined as follows: We move $S^{1}(S)$ and $S_{\text {eq }}^{1}$ by small isometries on $\mathbb{C}$ and $S^{2}(1)$ respectively and obtain $S_{2}^{1}(S), S_{2 \text {,eq }}^{1}$ so that

$$
\psi_{H}\left(L^{(0)}\right) \cap\left(L^{(1)}\right)=\emptyset
$$

where we denote $L^{(1)}:=S_{2}^{1}(S) \times S_{2, \text { eq }}^{1}$.

Since the condition (3.1) is an open condition, such an isometry obviously exists. We choose this perturbation so that

$$
\operatorname{dist}\left(L^{(0)}, L^{(1)}\right)=\operatorname{dist}\left(S^{1}(S) \times S_{\text {eq }}^{1}, S_{2}^{1}(S) \times S_{2, \text { eq }}^{1}\right) \leq \frac{c}{4} .
$$

We then will deduce contradiction. We remark that $L^{(0)} \cap L^{(1)}$ consists of four transversal intersection points. We take a one-parameter family of smooth functions $\chi_{K}$ on $\mathbb{R}$ satisfying (2.5). We denote by $X_{H}$ the Hamiltonian vector filed of $H$ defined by $d H=\omega\left(X_{H}, \cdot\right)$.

Consider any pair $\left(p_{-}, p_{+}\right)$of intersection points in $L^{(0)} \cap L^{(1)}$ and the equation

$$
\frac{\partial u}{\partial \tau}+J\left(\frac{\partial u}{\partial t}-\chi_{K}(\tau) X_{H}(u)\right)=0
$$

of $u=u(\tau, t): \mathbb{R} \times[0,1] \rightarrow \mathbb{C} \times S^{2}$ satisfying the boundary condition

$$
u(\tau, 0) \in L^{(0)}, u(\tau, 1) \in L^{(1)}, u( \pm \infty, t) \equiv p_{ \pm} \in L^{(0)} \cap L^{(1)}
$$

and the finite energy condition

$$
E_{\left(J, H, \chi_{K}\right)}(u):=\frac{1}{2} \int_{\mathbb{R} \times[0,1]}\left|\frac{\partial u}{\partial \tau}\right|^{2}+\left|\frac{\partial u}{\partial t}-\chi_{K}(\tau) X_{H}(u)\right|^{2} d \tau d t<\infty .
$$

Here we use the canonical complex structure $J$ on $\mathbb{C} \times S^{2}(1)$, which we do not perturb. Note that any solution $u$ of $(3.3),(3.4)$ carries a natural homotopy class. We denote this homotopy class by $B$. As standard in Floer theory we define the equivalence relation $B \sim B^{\prime}$ if and only if

$$
\omega\left(B_{1}\right)=\omega\left(B_{2}\right), \quad \mu\left(B_{1}\right)=\mu\left(B_{2}\right)
$$

and denote by $\Pi\left(p_{-}, p_{+}\right)$the set of equivalence classes. Here $\mu$ denotes the Maslov index of the map $u$ associated to the pairs $\left(L^{(0)}, L^{(1)}\right)$ of Lagrangian submanifolds and the asymptotic condition $p_{ \pm}$. (We refer to Section $2.2[\mathbf{3}]$ for a complete discussion on the homotopy class and the Novikov covering.)

The following energy estimate will be proved in Section 5. See Proposition 5.4.

Lemma 3.1. Let $0 \leq K<\infty$ and $u$ be any finite energy solution of (3.3), (3.4). Then

$$
E_{\left(J, H, \chi_{K}\right)}(u) \leq \int u^{*} \omega+\|H\|
$$


We consider the parameterized moduli space

$$
\mathcal{M}^{\text {para }}\left(p_{-}, p_{+} ; B\right)=\bigcup_{K \in \mathbb{R}_{\geq 0}}\{K\} \times \mathcal{M}^{K}\left(p_{-}, p_{+} ; B\right)
$$

where $\mathcal{M}^{K}\left(p_{-}, p_{+} ; B\right)$ is the space of solutions in class $B$ to equation (3.3) satisfying the conditions (3.4) and (3.5) for the parameter $K \in[0, \infty)$. We note that the symplectic area $\int_{\mathbb{R} \times[0,1]} u^{*} \omega$ is invariant under the homotopy in the space of smooth maps with the boundary condition (3.4), which is fixed. We shall consider only the triples $\left(p_{-}, p_{+} ; B\right)$ whose associated moduli space $\mathcal{M}^{\text {para }}\left(p_{-}, p_{+} ; B\right)$ has virtual dimension 0 or 1 .

To study $\mathcal{M}\left(p_{-}, p_{+} ; B\right)=\mathcal{M}^{K=0}\left(p_{-}, p_{+} ; B\right)$, we also need to study the equation

$$
\frac{\partial u}{\partial \tau}+J \frac{\partial u}{\partial t}=0
$$

with the same fixed Lagrangian boundary condition but possibly with different asymptotic condition $\left(p_{-}^{\prime}, p_{+}^{\prime}\right)$. The associated moduli space carries the natural $\mathbb{R}$-action and denote by

$$
\overline{\mathcal{M}}\left(p_{-}^{\prime}, p_{+}^{\prime} ; B^{\prime}\right)
$$

the compactification of its quotient by this $\mathbb{R}$-action. We shall however need to consider only those $\left(p_{-}^{\prime}, p_{+}^{\prime} ; B^{\prime}\right)$ whose associated moduli space has virtual dimension 0.

Note that all the non-trivial holomorphic disk which bounds either $L^{(0)}$ or $L^{(1)}$ have Maslov index $\geq 2$ and all the holomorphic spheres have Chern number $\geq 2$. Hence we can perturb the data such as Hamiltonians so that the moduli spaces $\mathcal{M}^{\text {para }}\left(p_{-}, p_{+} ; B\right)$ of negative virtual dimension are empty. Then the moduli spaces of virtual dimension 0 or 1 do not have holomorphic disk bubbles nor holomorphic sphere bubbles. We would like to point out that the necessary transversality result on the moduli spaces can be easily achieved in the current context: For $\mathcal{M}^{\text {para }}\left(p_{-}, p_{+}\right)$we can perturb Hamiltonian term on compact set to make it transversal. For $\overline{\mathcal{M}}\left(p_{-}, p_{+}\right)$we can directly check that this moduli space is transversal using the fact that our chosen almost complex structure $J$ is the standard integrable one on $\mathbb{C} \times S^{2}(1)$.

Now we study the end and the boundary of $\mathcal{M}^{\text {para }}(p, p ; 0)$ for a $p \in$ $L^{(0)} \cap L^{(1)}$. The space $\mathcal{M}^{\text {para }}(p, p ; 0)$ is one-dimensional. Here, $0 \in \Pi(p, p)$ denotes the equivalence class corresponding to the constant map $u \equiv p$. In particular, we have

$$
\int u^{*} \omega=0 .
$$


We observe that the condition $\psi_{H}\left(L^{(0)}\right) \cap L^{(1)}=\emptyset$ implies the following

Lemma 3.2. For all sufficiently large $K>0,(3.3)$ has no solution. Namely, we have $\mathcal{M}^{K}(p, p ; 0)=\emptyset$.

Proof. Suppose that there is a sequence $K_{i}$ such that $u_{i} \in \mathcal{M}^{K_{i}}(p, p ; 0)$ and $K_{i} \rightarrow+\infty$. Note that we already excluded bubbling-off of $J$-holomorphic spheres and discs from the one-dimensional moduli space $\mathcal{M}^{\text {para }}(p, p ; 0)$. Since $\left\|\frac{\partial u_{i}}{\partial \tau}\right\| \leq\|H\|$ by (3.7) in Lemma 3.1 and (3.8), we find that $u_{i}$ converges to some $u_{\infty}$ uniformly on compact sets in $\mathbb{R} \times[0,1]$. By taking a limit as $\tau \rightarrow \pm \infty, u_{\infty}$ converges in compact open topology to a $\tau$-independent map $\gamma(t)$, which satisfies

$$
\frac{d \gamma(t)}{d t}=X_{H_{t}}(\gamma(t))
$$

But it implies that $\psi_{H}\left(L^{(0)}\right) \cap L^{(1)} \neq \emptyset$. This is a contradiction.

So the boundary of the compactified moduli space

$$
\overline{\mathcal{M}}^{\text {para }}(p, p ; 0)
$$

consist of the following three types:

(1) $K=0$.

$$
\bigcup_{q} \overline{\mathcal{M}}\left(p, q ; B_{1}\right) \times \mathcal{M}^{\text {para }}\left(q, p ; B_{2}\right), B_{1}+B_{2}=0
$$

$$
\bigcup_{q} \mathcal{M}^{\mathrm{para}}\left(p, q ; B_{1}\right) \times \overline{\mathcal{M}}\left(q, p: B_{2}\right), B_{1}+B_{2}=0 .
$$

The case (1) gives rise to exactly one element. (That is the constant map, $u \equiv p$.) Therefore the sum of the numbers of the boundaries of type (3.9) and of (3.10) must be odd. We will show that this is impossible.

For this purpose, we examine each of the two types in detail. We first consider the case of (3.9). Let

$$
\left(v,\left(u, K_{0}\right)\right) \in \overline{\mathcal{M}}\left(p, q ; B_{1}\right) \times \mathcal{M}^{\mathrm{para}}\left(q, p ; B_{2}\right) .
$$

The energy bound (3.7) and (3.5) yield the inequality

$$
0 \leq E_{\left(J, H ; \chi_{K}\right)}(u) \leq \int u^{*} \omega+\|H\|
$$

for any solution of (3.3) for any $K$. Therefore from $\|H\|=S-c<S$ and (3.2), we derive

$$
\int u^{*} \omega \geq-\|H\|=-S+c
$$


Since we consider an element $v$ of $\overline{\mathcal{M}}\left(p, q ; B_{1}\right)$ whose virtual dimension is 0 , the element $v$ must be of the product form into

(a) $\left(v_{1},\{p t\}\right)$ with $v_{1}: \mathbb{R} \times[0,1] \rightarrow \mathbb{C}$ holomorphic,

(b) $\left(\{p t\}, v_{2}\right)$ with $v_{2}: \mathbb{R} \times[0,1] \rightarrow S^{2}(1)$ holomorphic.

First, consider the case (a). We note that there are three bounded connected components of $\mathbb{C} \backslash\left(S^{1}(S) \cup S_{2}^{1}(S)\right)$. Two of them say $D_{1}, D_{2}$ have small area and the third one $D_{3}$ has area $S-\epsilon$ for some $\epsilon>0$. By choosing a small isometry that we use in the beginning to define $L_{1}$, we may choose $\epsilon \leq \frac{c}{3}$ so that

$$
\epsilon+\frac{c}{3}+\|H\|<S-\frac{c}{3} .
$$

For this choice of $\epsilon>0$, we claim $D_{3}$ cannot appear in the compactification of $\mathcal{M}^{\text {para }}(p, p ; 0)$. In fact, if it did, $u \in \mathcal{M}^{\text {para }}\left(q, p ; B_{2}\right)$ and $D_{3}$ would give an element of this compactification that lead to

$$
0=\int u^{*} \omega+\int v^{*} \omega \geq-S+c+\operatorname{Area}\left(D_{3}\right) \geq-S+c+S-\frac{c}{3}=\frac{2 c}{3}>0,
$$

a contradiction.

Note that $D_{1}$ and $D_{2}$ have the same area $\int_{D_{i}} \omega=\omega\left(B_{1}\right)$. Therefore the end of $\mathcal{M}^{\text {para }}(q, p ; 0)$ will come in a pair of the form $\left(v_{ \pm},\left(u, K_{0}\right)\right)$ contained in

$$
\overline{\mathcal{M}}\left(p, q ; B_{1}\right) \times \mathcal{M}^{\text {para }}\left(q, p ; B_{2}\right)
$$

so that for each given $\left(u, K_{0}\right) \in \mathcal{M}^{\text {para }}\left(q, p ; B_{2}\right)$, there is a pair $v_{-}, v_{+} \in$ $\overline{\mathcal{M}}\left(p, q ; B_{1}\right)$. Therefore the cardinality of this set must be even.

For the case (b), similarly the end element again comes in a pair of the form $\left(\left(u, K_{0}\right), v_{ \pm}\right)$with the the same area $\int v_{-}^{*} \omega=\int v_{+}^{*} \omega$. (Here we use the fact that we put the equator on $S^{2}(1)$.) Again the cardinality of this set is even.

By the same argument, (3.10) consists of even number of points. This is a contradiction.

\section{Comparison of two Cauchy-Riemann equations and intertwining transformation}

In this section, we explain what the intertwining transformation we called in the introduction means. To describe it precisely, we briefly recall the Novikov covering spaces of the path spaces joining Lagrangian submanifolds, on which the action functional will be defined.

We denote the path space by

$$
\Omega\left(L^{(0)}, L^{(1)}\right)=\left\{\ell:[0,1] \rightarrow X \mid \ell(0) \in L^{(0)}, \ell(1) \in L^{(1)}\right\} .
$$


We first recall the action one-form $\alpha$ on $\Omega\left(L^{(0)}, L^{(1)}\right)$ defined by

$$
\alpha(\ell)(\xi)=\int_{0}^{1} \omega(\dot{\ell}(t), \xi(t)) d t
$$

This form is a 'closed' one-form but not 'exact' in general. Due to the presence of the multi-valuedness of the associated action functional we consider the Novikov covering space $\widetilde{\Omega}\left(L^{(0)}, L^{(1)} ; \ell_{a}\right)$ of the connected component $\Omega\left(L^{(0)}, L^{(1)} ; \ell_{a}\right)$ containing a chosen base path $\ell_{a} \in \Omega\left(L^{(0)}, L^{(1)}\right)$ for each

$$
a \in \pi_{0}\left(\Omega\left(L^{(0)}, L^{(1)}\right)\right),
$$

and consider its associated action functional

$$
\mathcal{A}_{\ell_{a}}([\ell, w])=\int w^{*} \omega \quad \text { for } \quad[\ell, w] \in \widetilde{\Omega}\left(L^{(0)}, L^{(1)} ; \ell_{a}\right) .
$$

A simple computation shows that

$$
d \mathcal{A}_{\ell a}=-\pi^{*} \alpha
$$

on $\widetilde{\Omega}\left(L^{(0)}, L^{(1)} ; \ell_{a}\right)$. Here $[\ell, w] \in \widetilde{\Omega}\left(L^{(0)}, L^{(1)} ; \ell_{a}\right)$ is an equivalence class of the pair $(\ell, w)$ of $\ell \in \Omega\left(L^{(0)}, L^{(1)} ; \ell_{a}\right)$ and $w:[0,1]^{2} \rightarrow X$ satisfying

$$
w(s, 0) \in L^{(0)}, w(s, 1) \in L^{(1)}, w(0, t)=\ell_{a}(t), w(1, t)=\ell(t)
$$

and $\pi: \widetilde{\Omega}\left(L^{(0)}, L^{(1)} ; \ell_{a}\right) \rightarrow \Omega\left(L^{(0)}, L^{(1)}\right)$ is the natural projection given by $[\ell, w] \mapsto \ell$. The equivalence relation is given as follows; $\left(\ell, w_{1}\right) \sim\left(\ell, w_{2}\right)$ if and only if

$$
\omega\left(\left[\bar{w}_{1} \# w_{2}\right]\right)=0=\mu_{L^{(0)} L^{(1)}}\left(\bar{w}_{1} \# w_{2}\right)
$$

where $\mu_{L^{(0)} L^{(1)}}$ is the Maslov index of the annulus map $\bar{w} \# w^{\prime}: S^{1} \times[0,1] \rightarrow$ $X$ with boundary lying on $L^{(0)}$ at $t=0$ and on $L^{(1)}$ at $t=1$. We refer to Definition 2.2.4 [3] for the precise definition of $\widetilde{\Omega}\left(L^{(0)}, L^{(1)} ; \ell_{a}\right)$.

Remark 4.1. In this section and the next, we pick and discuss one connected component of $\Omega\left(L^{(0)}, L^{(1)}\right)$ and its Novikov covering space without loss of generality. In Section 6, we will consider all connected components to study Floer chain complex which is, in fact, the direct sum of Floer chain complex for each connected component.

Now for a pair $\left(L^{(0)}, L^{(1)}\right)$ of compact Lagrangian submanifolds we consider the Hamiltonian deformation $\left(L^{(0) \prime}, L^{(1) \prime}\right)$ given by

$$
L^{(0) \prime} \in \mathfrak{I} \mathfrak{s o}\left(L^{(0)}\right), \quad L^{(1) \prime} \in \mathfrak{I} \mathfrak{s o}\left(L^{(1)}\right) .
$$

We also consider a family $J^{s}=\left\{J_{t}^{s}\right\}_{0 \leq t \leq 1}$ of $\omega$-compatible almost complex structures. We take Hamiltonian isotopies $\phi_{H^{(0)}}=\left\{\phi_{H^{(0)}}^{s}\right\}_{0 \leq s \leq 1}, \phi_{H^{(1)}}=$ $\left\{\phi_{H^{(1)}}^{s}\right\}_{0 \leq s \leq 1}$ such that

$$
\phi_{H^{(0)}}^{1}\left(L^{(0)}\right)=L^{(0) \prime}, \quad \phi_{H^{(1)}}^{1}\left(L^{(1)}\right)=L^{(1) \prime} .
$$


Set

$$
\psi^{t}=\phi_{H^{(0)}}^{t} \circ\left(\phi_{H^{(0)}}^{1}\right)^{-1} \circ \phi_{H^{(1)}}^{1-t} \circ\left(\phi_{H^{(1)}}^{1}\right)^{-1} .
$$

Note that $\psi^{0}=\left(\phi_{H^{(0)}}^{1}\right)^{-1}, \psi^{1}=\left(\phi_{H^{(1)}}^{1}\right)^{-1}$ and

$$
\frac{d}{d t} \psi^{t}(p)=X_{\widetilde{H}_{t}}\left(\psi^{t}(p)\right)
$$

where

$$
\widetilde{H}_{t}=H_{t}^{(0)}-H_{1-t}^{(1)} \circ \phi_{H^{(0)}}^{1} \circ\left(\phi_{H^{(0)}}^{t}\right)^{-1} .
$$

We may also write $\widetilde{H}(t, x)=\widetilde{H}_{t}(x)$. Set

$$
\ell_{a}^{\prime}(t)=\left(\psi^{t}\right)^{-1}\left(\ell_{a}(t)\right)
$$

and define a bijective correspondence

$$
\ell(t) \in \Omega\left(L^{(0)}, L^{(1)}: \ell_{a}\right) \mapsto\left(\psi^{t}\right)^{-1} \circ \ell(t) \in \Omega\left(L^{(0) \prime}, L^{(1) \prime} ; \ell_{a}^{\prime}\right) .
$$

The main goal of Sections 4-6 is as follows. We first obtain an appropriate estimate for solutions of the Hamiltonian perturbed Cauchy-Riemann equation (4.14). We use it to construct a filtered $A_{\infty}$-bimodule homomorphism. It then induces a chain map between Floer complexes when the pair $\left(L^{(0)}, L^{(1)}\right)$ is unobstructed in the sense of Lagrangian Floer cohomology theory,

$$
C\left(L^{(1)}, L^{(0)} ; \Lambda_{\text {nov }}\right) \rightarrow C\left(L^{(1) \prime}, L^{(0) \prime} ; \Lambda_{\text {nov }}\right) .
$$

Our concern is the filtration change. See the beginning of Section 6 for a quick review of $C\left(L^{(1)}, L^{(0)} ; \Lambda_{\text {nov }}\right)$. For the construction of the filtered $A_{\infty}$-bimodule homomorphism, see the Appendix.

The reason why we use equation (4.14) instead of the Cauchy-Riemann equation with moving Lagrangian boundary condition (4.19) to construct a desired weakly filtered $A_{\infty}$ bimodule homomorphism is to apply the improved estimates for solutions of (4.14) carried out in Section 5.

We would like to compare the geometric version of Floer theory and the dynamical one. Such a comparison is by now well-known, and, for example, the case

$$
\left(L^{(0)}, L^{(1)}\right), \quad\left(\phi_{H}^{1}\left(L^{(0)}\right), L^{(1)}\right)
$$

was exploited previously in [11] for the exact Lagrangian submanifolds on the cotangent bundle in relation to the study of spectral invariants. Here we need such a study for general compact Lagrangian submanifolds on general $(X, \omega)$.

The geometric version of the Floer complex for $\left(L^{(0) \prime}=\phi_{H^{(0)}}^{1}\left(L^{(0)}\right)\right.$, $\left.L^{(1) \prime}=\phi_{H^{(1)}}^{1}\left(L^{(1)}\right)\right)$ is generated by the intersection points

$$
\phi_{H^{(0)}}^{1}\left(L^{(0)}\right) \cap \phi_{H^{(1)}}^{1}\left(L^{(1)}\right)
$$


and its Floer boundary map is constructed by the moduli space of genuine Cauchy-Riemann equation

$$
\left\{\begin{array}{l}
\frac{\partial u^{\prime}}{\partial \tau}+J^{\prime} \frac{\partial u^{\prime}}{\partial t}=0, \\
u^{\prime}(\tau, 0) \in L^{(0) \prime}, \quad u^{\prime}(\tau, 1) \in L^{(1) \prime} .
\end{array}\right.
$$

Here $J^{\prime}=J_{t}^{\prime}=\left(\psi^{t}\right)_{*}^{-1} \circ J_{t} \circ\left(\psi^{t}\right)_{*}$, where $\psi^{t}$ is given in (4.6). We denote by $\mathcal{M}\left(\phi_{H^{(1)}}^{1}\left(L^{(1)}\right), \phi_{H^{(0)}}^{1}\left(L^{(0)}\right) ; J^{\prime}\right)$ the moduli space of finite energy solutions of this equation. Owing to the presence of the multi-valuedness of the associated action functional, we need to consider these equations on the Novikov covering space of some specified connected component

$$
\Omega\left(\phi_{H^{(0)}}^{1}\left(L^{(0)}\right), \phi_{H^{(1)}}^{1}\left(L^{(1)}\right) ; \ell_{a}^{\prime}\right)
$$

with the base path $\ell_{a}^{\prime}(t)=\left(\psi^{t}\right)^{-1} \circ \ell_{a}(t)$ and consider the action functional

$$
\mathcal{A}_{\ell_{a}^{\prime}}\left(\left[\ell^{\prime}, w^{\prime}\right]\right)=\int\left(w^{\prime}\right)^{*} \omega
$$

where $\left[\ell^{\prime}, w^{\prime}\right] \in \widetilde{\Omega}\left(\phi_{H^{(0)}}^{1}\left(L^{(0)}\right), \psi_{H^{(1)}}^{1}\left(L^{(1)}\right) ; \ell_{a}^{\prime}\right)$ and $w^{\prime}:[0,1]^{2} \rightarrow X$ is a map satisfying the boundary condition

$$
\begin{aligned}
w^{\prime}(0, t) & =\ell_{a}^{\prime}(t), w^{\prime}(1, t)=\ell^{\prime}(t), w^{\prime}(s, 0) \in \phi_{H^{(0)}}^{1}\left(L^{(0)}\right), \\
w^{\prime}(s, 1) & \in \phi_{H^{(1)}}^{1}\left(L^{(1)}\right) .
\end{aligned}
$$

On the other hand, the dynamical version of the Floer complex is generated by the solutions of Hamilton's equation

$$
\dot{x}=X_{\widetilde{H}_{t}}(x), \quad x(0) \in L^{(0)}, x(1) \in L^{(1)}
$$

and its boundary map is constructed by the moduli space of perturbed Cauchy-Riemann equation

$$
\left\{\begin{array}{l}
\frac{\partial u}{\partial \tau}+J\left(\frac{\partial u}{\partial t}-X_{\widetilde{H}_{t}}(u)\right)=0 \\
u(\tau, 0) \in L^{(0)}, \quad u(\tau, 1) \in L^{(1)}
\end{array}\right.
$$

We denote by $\mathcal{M}\left(L^{(1)}, L^{(0)} ; \widetilde{H} ; J\right)$ the moduli space of finite energy solutions of this equation. The action functional $\mathcal{A}_{\widetilde{H}, \ell_{a}}$ is defined by

$$
\mathcal{A}_{\widetilde{H}, \ell_{a}}([\ell, w])=\int w^{*} \omega+\int_{0}^{1} \widetilde{H}(t, \ell(t)) d t
$$

on $\widetilde{\Omega}\left(L^{(0)}, L^{(1)} ; \ell_{a}\right)$. 
These two Floer theories are related by the following transformations of the bijective map:

$$
\begin{gathered}
\mathfrak{g}_{H^{(0)}, H^{(1)}}^{+}: \widetilde{\Omega}\left(\phi_{H^{(0)}}^{1}\left(L^{(0)}\right), \phi_{H^{(1)}}^{1}\left(L^{(1)}\right) ; \ell_{a}^{\prime}\right) \rightarrow \widetilde{\Omega}\left(L^{(0)}, L^{(1)} ; \ell_{a}\right), \\
{\left[\ell^{\prime}, w^{\prime}\right] \mapsto[\ell, w]}
\end{gathered}
$$

given by the assignment

$$
\ell(t)=\psi^{t}\left(\ell^{\prime}(t)\right), \quad w(s, t)=\psi^{t}\left(w^{\prime}(s, t)\right)
$$

This provides a bijective correspondence of the critical points

$$
\operatorname{Crit} \mathcal{A}_{\ell_{a}^{\prime}} \longleftrightarrow \operatorname{Crit} \mathcal{A}_{\widetilde{H}, \ell} ; \quad\left[p^{\prime}, w^{\prime}\right] \mapsto\left[z_{p^{\prime}}^{\widetilde{H}}, w\right],
$$

where $p^{\prime} \in \phi_{H^{(0)}}^{1}\left(L^{(0)}\right) \cap \phi_{H^{(1)}}^{1}\left(L^{(1)}\right)$ and we set

$$
z_{p^{\prime}}^{\widetilde{H}}(t):=\psi^{t}\left(p^{\prime}\right), \quad w:=\psi^{t}\left(w^{\prime}(s, t)\right) .
$$

We also have an isomorphism between the moduli spaces

$$
\mathcal{M}\left(\phi_{H^{(1)}}^{1}\left(L^{(1)}\right), \phi_{H^{(0)}}^{1}\left(L^{(0)}\right) ; J^{\prime}\right) \rightarrow \mathcal{M}\left(L^{(1)}, L^{(0)} ; \widetilde{H} ; J\right)
$$

with $J_{t}=\psi_{*}^{t} \circ J_{t}^{\prime} \circ\left(\psi_{*}^{t}\right)^{-1}$ where the map is defined by

$$
u(\tau, t)=\psi^{t}\left(u^{\prime}(\tau, t)\right) .
$$

The map $\mathfrak{g}_{H^{(0)}, H^{(1)}}^{+}$also preserves the action up to a constant in that

Lemma 4.2. Denote

$$
c\left(\widetilde{H} ; \ell_{a}\right):=\int_{0}^{1} \widetilde{H}\left(t, \ell_{a}(t)\right) d t,
$$

which is a constant depending only on $\widetilde{H}$ and the base path $\ell_{a}$ of the connected component $\Omega\left(L^{(0)}, L^{(1)} ; \ell_{a}\right)$. Then

$$
\mathcal{A}_{\widetilde{H}, \ell_{a}} \circ \mathfrak{g}_{H^{(0)}, H^{(1)}}^{+}\left(\left[\ell^{\prime}, w^{\prime}\right]\right)=\mathcal{A}_{\ell_{a}^{\prime}}\left(\left[\ell^{\prime}, w^{\prime}\right]\right)+c\left(\widetilde{H} ; \ell_{a}\right)
$$

on $\widetilde{\Omega}\left(L^{(0)}, L^{(1)} ; \ell_{a}\right)$.

Remark 4.3. Since we normalized Hamiltonians so that $\int_{X} H_{t}^{(i)} \omega^{n}=0$ for each $t$ and $i=0,1$ in Section 2, hence we also have $\int_{X} \widetilde{H}_{t} \omega^{n}=0$, we can take $\ell_{a}$ for each connected component of $\Omega\left(L^{(0)}, L^{(1)}\right)$ in such a way that $c\left(\widetilde{H} ; \ell_{a}\right)=\int_{0}^{1} \widetilde{H}\left(t, \ell_{a}(t)\right) d t=0$. It is not essential to choose $\ell_{a}$ in a way as above. In fact, if we take a based path so that $c\left(\widetilde{H} ; \ell_{a}\right) \neq 0$, it is enough to include an extra term $c\left(\widetilde{H} ; \ell_{a}\right)$ in the energy estimate on $\Omega\left(L^{(0)}, L^{(1)} ; \ell_{a}\right)$ when we apply the intertwining transformation $\mathfrak{g}_{H^{(0)}, H^{(1)}}^{+}$or its inverse. Since we will consider all connected components of $\Omega\left(L^{(0)}, L^{(1)}\right)$ in Section 6 (see Remark 4.1), we have to add the constant $c\left(\widetilde{H} ; \ell_{a}\right)$ for each connected component $\Omega\left(L^{(0)}, L^{(1)} ; \ell_{a}\right)$. Thus, to avoid heavy notation, we simply choose $\ell_{a}$ so that $c\left(\widetilde{H} ; \ell_{a}\right)=0$ for each connected component $\Omega\left(L^{(0)}, L^{(1)} ; \ell_{a}\right)$. 
Proof. Let $\left[\ell^{\prime}, w^{\prime}\right] \in \widetilde{\Omega}\left(\phi_{H^{(0)}}^{1}\left(L^{(0)}\right), \phi_{H^{(1)}}^{1}\left(L^{(1)}\right) ; \ell_{a}^{\prime}\right)$. Then we have

$$
\mathcal{A}_{\widetilde{H} ; \ell_{a}}\left(\mathfrak{g}_{H^{(0)}, H^{(1)}}^{+}\left(\left[\ell^{\prime}, w^{\prime}\right]\right)\right)=\int w^{*} \omega+\int_{0}^{1} \widetilde{H}(t, \ell(t)) d t
$$

and

We compute

$$
w^{*} \omega=\omega\left(\frac{\partial w}{\partial s}, \frac{\partial w}{\partial t}\right) d s \wedge d t
$$

$$
\begin{aligned}
& \frac{\partial w}{\partial s}=d \psi^{t}\left(\frac{\partial w^{\prime}}{\partial s}\right), \\
& \frac{\partial w}{\partial t}=d \psi^{t}\left(\frac{\partial w^{\prime}}{\partial t}\right)+X_{\widetilde{H}_{t}}(w(s, t)) .
\end{aligned}
$$

Substituting this into the above, we obtain

$$
\begin{aligned}
\int w^{*} \omega= & \int_{0}^{1} \int_{0}^{1} \omega\left(\frac{\partial w}{\partial s}, \frac{\partial w}{\partial t}\right) d s d t \\
= & \int_{0}^{1} \int_{0}^{1} \omega\left(\frac{\partial w^{\prime}}{\partial s}, \frac{\partial w^{\prime}}{\partial t}\right) d s d t \\
& \quad+\int_{0}^{1} \int_{0}^{1} \omega\left(d \psi^{t}\left(\frac{\partial w^{\prime}}{\partial s}\right), X_{\widetilde{H}_{t}}(w(s, t))\right) d s d t \\
= & \int\left(w^{\prime}\right)^{*} \omega-\int_{0}^{1} \int_{0}^{1} d \widetilde{H}_{t}(w(s, t))\left(d \psi^{t} \frac{\partial w^{\prime}}{\partial s}\right) d s d t \\
= & \int\left(w^{\prime}\right)^{*} \omega-\int_{0}^{1} \int_{0}^{1} \frac{\partial}{\partial s} \widetilde{H}_{t}(w(s, t)) d s d t \\
= & \int\left(w^{\prime}\right)^{*} \omega-\int_{0}^{1} \widetilde{H}_{t}(w(1, t)) d t+\int_{0}^{1} \widetilde{H}_{t}(w(0, t)) d t .
\end{aligned}
$$

Substituting this into the above definition of $\mathcal{A}_{\widetilde{H} ; \ell_{a}}\left(\mathfrak{g}_{H^{(0)}, H^{(1)}}^{+}\left(\left[\ell^{\prime}, w^{\prime}\right]\right)\right)$, the proof is finished.

We denote by $\mathfrak{g}_{H^{(0)}, H^{(1)}}^{-}$the inverse $\left(\mathfrak{g}_{H^{(0)}, H^{(1)}}^{+}\right)^{-1}$. The outcome of the above discussion is that the two associated Floer cohomologies are isomorphic to each other.

Remark 4.4. For a pair $\left(L^{(0)}, L^{(1)}\right)$ of compact Lagrangian submanifolds we consider the Hamiltonian deformation $\left(L^{(0) \prime}, L^{(1) \prime}\right)$ given by

$$
L^{(0) \prime} \in \mathfrak{I} \mathfrak{s o}\left(L^{(0)}\right), \quad L^{(1) \prime} \in \mathfrak{I} \mathfrak{s o}\left(L^{(1)}\right) .
$$

In order to construct a chain homomorphism between $C\left(L^{(1)}, L^{(0)} ; \Lambda\right)$ and $C\left(L^{(1) \prime}, L^{(0) \prime} ; \Lambda\right)$, we often use the moduli spaces of solutions of the CauchyRiemann equation with moving Lagrangian boundary conditions, e.g., [10], as below. 
We consider a family $J^{s}=\left\{J_{t}^{s}\right\}_{0 \leq t \leq 1}$ of $\omega$-compatible almost complex structures. We take Hamiltonian isotopies $\phi_{H^{(0)}}=\left\{\phi_{H^{(0)}}^{s}\right\}_{0 \leq s \leq 1}, \phi_{H^{(1)}}=$ $\left\{\phi_{H^{(1)}}^{s}\right\}_{0 \leq s \leq 1}$ as in (4.5). For a given pair of Hamiltonian isotopies $\phi_{H^{(i)}}$ for $i=0,1, J^{s}=\left\{J_{t}^{s}\right\}$, and a given smooth function $\chi$ as in Section 2, we consider moving Lagrangian boundary value problem

$$
\left\{\begin{array}{l}
\frac{\partial u}{\partial \tau}+J^{\chi} \frac{\partial u}{\partial t}=0 \\
u(\tau, 0) \in \phi_{H^{(0)}}^{\chi(\tau)}\left(L^{(0)}\right), u(\tau, 1) \in \phi_{H^{(1)}}^{\chi(\tau)}\left(L^{(1)}\right)
\end{array}\right.
$$

where $J^{\chi}(\tau, t)=J_{t}^{\chi(\tau)}$. Let $\left[\ell_{p}, w\right] \in \widetilde{\Omega}\left(L^{(0)}, L^{(1)} ; \ell_{a}\right)$, resp. $\left[\ell_{q^{\prime}}, w^{\prime}\right] \in$ $\widetilde{\Omega}\left(L^{(0) \prime}, L^{(1) \prime} ; \ell_{a}^{\prime}\right)$. Here we choose $\ell_{a}^{\prime}(t)$ as the base path of $\Omega\left(L^{(0) \prime}, L^{(1) \prime}\right)$ given in (4.9). See also the bijective correspondence (4.16). (We used the notation $\ell_{0}^{\left(\psi^{(0)}, \psi^{(1)}\right)}$ in $[\mathbf{3}]$.) We denote by

$$
\mathcal{M}^{\chi}\left(\left(L^{(1)}, \phi_{H^{(1)}}^{1}\right),\left(L^{(0)}, \phi_{H^{(0)}}^{1}\right) ;\left[\ell_{p}, w\right],\left[\ell_{q^{\prime}}, w^{\prime}\right]\right)
$$

the set of solutions of (4.19) with

$$
\left[\ell_{q^{\prime}}, I_{\phi_{H^{(0)}}^{1}, \phi_{H^{(1)}}^{1}}^{\chi}(w \# u)\right]=\left[\ell_{q^{\prime}}, w^{\prime}\right]
$$

where $w \# u:[0,1] \times[0,1] \rightarrow X$ is the concatenation in the $\tau$-direction of $w$ and $u$ and

$$
I_{\phi_{H^{(0)}}^{1}, \phi_{H^{(1)}}^{1}}^{\chi} v(\tau, t)=\left(\phi_{H^{(1)}}^{1} \circ\left(\phi_{H^{(1)}}^{\chi(\tau)(1-t)}\right)^{-1} \circ \phi_{H^{(0)}}^{1} \circ\left(\phi_{H^{(0)}}^{\chi(\tau) t}\right)^{-1}\right) v(\tau, t) .
$$

(see p. 308 in [3]). We note that we do not use equation (4.19) of moving Lagrangian boundary value problem and the moduli space (4.20) themselves in this paper, while in Section 5.3.2 of [3] we used them for construction of a weakly filtered $A_{\infty}$ bimodule homomorphism.

Remark 4.5. In Proposition 5.3.20 (restated as Proposition 5.3.45) in [3], we claimed that

$$
\mathcal{A}_{\ell_{0}^{\left(\psi^{(0)}, \psi^{(1)}\right)}}\left(\left[\ell_{q}, w^{\prime}\right]\right)-\mathcal{A}_{\ell_{0}}\left(\left[\ell_{p}, w\right]\right)+\mathfrak{E} \geq 0
$$

with $\mathfrak{E}=\left\|\left\{\psi_{t}^{(0)}\right\}_{t}\right\|+\left\|\left\{\psi_{t}^{(1)}\right\}_{t}\right\|$, where $\left\|\left\{\psi_{t}^{(0)}\right\}_{t}\right\|,\left\|\left\{\psi_{t}^{(1)}\right\}_{t}\right\|$ are the Hofer lengths of Hamiltonian isotopies. (In the notation of this paper, they are denoted by leng $\left(\left\{\phi_{H^{(i)}}\right\}\right)=\left\|H^{(i)}\right\|, i=0,1$, when $\left\{\psi_{t}^{(i)}\right\}_{t}$ are generated by normalized Hamiltonians $H^{(i)}$.) However, (5.3.48) in [3] is not correct and should be replaced by Lemma 4.2 in this paper. Note that

$$
\int_{0}^{1} \min _{x \in X} \widetilde{H}(t, x) d t \leq \mathcal{A}_{\widetilde{H}, \ell_{a}}([\ell, w])-\mathcal{A}_{\ell_{a}}([\ell, w]) \leq \int_{0}^{1} \max _{x \in X} \widetilde{H}(t, x) d t .
$$


Hence, we have

$$
\left|c\left(\widetilde{H} ; \ell_{a}\right)-\left(\mathcal{A}_{\widetilde{H}, \ell_{a}}([\ell, w])-\mathcal{A}_{\ell_{a}}([\ell, w])\right)\right| \leq\|\widetilde{H}\| .
$$

As a conclusion, if $\left\{\psi_{t}^{(i)}\right\}_{t}$ is generated by normalized Hamiltonians $H^{(i)}$, $i=0,1$, we have

$$
\mathfrak{E}=2\left(\left\|\left\{\psi_{t}^{(0)}\right\}_{t}\right\|+\left\|\left\{\psi_{t}^{(1)}\right\}_{t}\right\|\right) .
$$

(Here we used that $\|\widetilde{H}\| \leq\left\|H^{(1)}\right\|+\left\|H^{(2)}\right\|$.) We used (4.21) to guarantee the energy estimate for the solutions of (4.11), which are used to define weakly filtered $A_{\infty}$-bimodule homomorphisms. For this purpose, it is enough to have (4.21) for some constant $\mathfrak{E}$. For the proof of Theorem 6.1.25 in [3], we need the improved estimate given in Proposition 5.6 for solutions of the perturbed Cauchy-Riemann equation (4.14) using Hamiltonians.

\section{Improved energy estimate}

Since we can perturb $L^{(0)}, L^{(1)}$ into transversal position by a Hamiltonian isotopy with arbitrary small Hofer energy, we assume that $L^{(0)}$ and $L^{(1)}$ are transversal from now on. First we consider the case of varying the first argument $L^{(0)}$ of the pair $\left(L^{(0)}, L^{(1)}\right)\left(L^{(0) \prime}, L^{(1) \prime}\right)=\left(\phi_{H^{(0)}}^{1}\left(L^{(0)}\right), \phi_{H^{(1)}}\left(L^{(1)}\right)\right)$. We can construct a chain homotopy equivalence using the moduli spaces of holomorphic strips with moving Lagrangian condition. However, as far as the study of the optimal filtration change is concerned, employing the moduli space with moving Lagrangian boundary is not the best one. We will show that employing the standard perturbed Cauchy-Riemann equation by Hamiltonian vector fields with fixed Lagrangian boundaries, which intertwines the geometric version and the dynamical version of the Floer complex, gives a stronger energy estimate which gives rise to the optimal change of filtration.

Let $\chi$ be one of smooth functions on $\mathbb{R}$ of the three types introduced in Section 2. See (2.4) and (2.5). Consider the perturbed Cauchy-Riemann equation (4.14):

$$
\left\{\begin{array}{l}
\frac{\partial u}{\partial \tau}+J\left(\frac{\partial u}{\partial t}-\chi(\tau) X_{\widetilde{H}}(u)\right)=0, \\
u(\tau, 0) \in L^{(0)}, u(\tau, 1) \in L^{(1)}
\end{array}\right.
$$

with the finite energy $E_{(J, \widetilde{H}, \chi)}(u)<\infty$. The following a priori energy bound is a key ingredient in relation to the lower bound of displacement energy. This kind of optimal estimate is originally due to Chekanov [1] , which is the key calculation that relates the energy and the Hofer norm in an optimal way. For completeness' sake, we include its proof which is a slight variation of the calculation carried out in p. 901 [12]. It is useful to decompose $\|\widetilde{H}\|$ 
into two parts

$$
E^{-}(\widetilde{H})=\int_{0}^{1}-\min \widetilde{H}_{t} d t, \quad E^{+}(\widetilde{H})=\int_{0}^{1} \max \widetilde{H}_{t} d t
$$

which are so-called the negative and positive part of the Hofer norm $\|\widetilde{H}\|$.

Lemma 5.1. Let $\chi=\chi_{+}$as in (2.4). Let $u$ be any finite energy solution of (4.14). Then we have

$$
\begin{aligned}
E_{(J, \widetilde{H}, \chi)}(u)= & \int u^{*} \omega+\int_{0}^{1} \widetilde{H}(t, u(\infty, t)) d t \\
& -\int_{-\infty}^{\infty} \chi^{\prime}(\tau) \int_{0}^{1}\left(\widetilde{H}_{t} \circ u\right) d t d \tau .
\end{aligned}
$$

Proof. The proof will be carried out by an explicit calculation. We compute

$$
\begin{aligned}
E_{(J, \widetilde{H}, \chi)}(u)=\int_{-\infty}^{\infty} \int_{0}^{1}\left|\frac{\partial u}{\partial \tau}\right|_{J}^{2} d t d \tau=\int_{-\infty}^{\infty} \int_{0}^{1} \omega\left(\frac{\partial u}{\partial \tau}, J \frac{\partial u}{\partial \tau}\right) d t d \tau \\
\quad=\int_{-\infty}^{\infty} \int_{0}^{1} \omega\left(\frac{\partial u}{\partial \tau}, \frac{\partial u}{\partial t}-\chi(\tau) X_{\widetilde{H}_{t}}(u)\right) d t d \tau \\
=\int_{-\infty}^{\infty} \int_{0}^{1} \omega\left(\frac{\partial u}{\partial \tau}, \frac{\partial u}{\partial t}\right) d t d \tau-\int_{-\infty}^{\infty} \chi(\tau) \int_{0}^{1} \omega\left(\frac{\partial u}{\partial \tau}, X_{\widetilde{H}_{t}(u)}\right) d t d \tau \\
=\int u^{*} \omega-\int_{-\infty}^{\infty} \chi(\tau) \int_{0}^{1}\left(-d \widetilde{H}_{t}(u) \frac{\partial u}{\partial \tau}\right) d t d \tau \\
=\int u^{*} \omega+\int_{-\infty}^{\infty} \chi(\tau) \int_{0}^{1} \frac{\partial}{\partial \tau}\left(\widetilde{H}_{t} \circ u\right) d t d \tau \\
=\int u^{*} \omega+\int_{0}^{1} \widetilde{H}(t, u(\infty, t)) d t-\int_{-\infty}^{\infty} \chi^{\prime}(\tau) \int_{0}^{1}\left(\widetilde{H}_{t} \circ u\right) d t d \tau .
\end{aligned}
$$

Here at the last equality, we do integration by parts and use the fact $\chi(\infty)=$ $1, \chi(-\infty)=0$. This completes the proof of (5.1).

This lemma gives rise to the following key formula of the action difference

$$
\mathcal{A}_{\widetilde{H}, \ell_{a}}(u(\infty))-\mathcal{A}_{\ell_{a}}(u(-\infty)) .
$$

Proposition 5.2. Let $p \in L^{(0)} \cap L^{(1)}$ and $q^{\prime} \in \phi_{H^{(0)}}^{1}\left(L^{(0)}\right) \cap \phi_{H^{(1)}}^{1}\left(L^{(1)}\right)$. Denote by $z_{q^{\prime}}^{\widetilde{H}} \in \Omega\left(L^{(0)}, L^{(1)} ; \ell_{a}\right)$ the Hamiltonian trajectory defined by

$$
z_{q^{\prime}}^{\widetilde{H}}(t)=\psi^{t}\left(q^{\prime}\right)
$$

and consider $\left[\ell_{p}, w\right] \in \operatorname{Crit} \mathcal{A}_{\ell_{a}},\left[z_{q^{\prime}}^{\widetilde{H}}, w^{\prime}\right] \in \operatorname{Crit} \mathcal{A}_{\widetilde{H}, \ell_{a}}$. Suppose that $u$ is any finite energy solution of (4.14) with $\chi=\chi_{+}$as in (2.4) satisfying the 
asymptotic condition and homotopy condition

$$
u(-\infty)=\ell_{p}, \quad u(\infty)=z_{q^{\prime}}^{\widetilde{H}}, \quad w \# u \sim w^{\prime} .
$$

Then we have

$$
\begin{aligned}
\mathcal{A}_{\widetilde{H}, \ell_{a}} & \left(\left[z_{q^{\prime}}^{\widetilde{H}}, w^{\prime}\right]\right)-\mathcal{A}_{\ell_{a}}\left(\left[w, \ell_{p}\right]\right) \\
& =E_{(J, \widetilde{H}, \chi)}(u)+\int_{-\infty}^{\infty} \int_{0}^{1} \chi^{\prime}(\tau) \widetilde{H}(t, u(\tau, t)) d t d \tau .
\end{aligned}
$$

Proof. By (5.2), we obtain

$$
\int u^{*} \omega=\int\left(w^{\prime}\right)^{*} \omega-\int w^{*} \omega .
$$

By substituting this into (5.1) and rearranging the resulting formula, we obtain (5.3) from the definitions (4.12) of $\mathcal{A}_{\ell_{a}}$ and (4.15) of $\mathcal{A}_{\widetilde{H}, \ell_{a}}$.

An immediate corollary is

Corollary 5.3. Suppose that there is a solution $u$ of (4.14) for $\chi=\chi_{+}$as in Proposition 5.2. Then we have

$$
\mathcal{A}_{\widetilde{H}, \ell_{a}}\left(\left[z_{q^{\prime}}^{\widetilde{H}}, w^{\prime}\right]\right)-\mathcal{A}_{\ell_{a}}\left(\left[\ell_{p}, w\right]\right) \geq \int_{0}^{1} \min \widetilde{H}_{t} d t=-E^{-}(\widetilde{H}) .
$$

Similarly, if there is a solution $u$ of (4.14) for $\chi_{-}=\chi_{-}=1-\chi_{+}$,

$$
\mathcal{A}_{\ell_{a}}\left(\left[\ell_{q}, w\right]\right)-\mathcal{A}_{\widetilde{H}, \ell_{a}}\left(\left[z_{p^{\prime}}^{\widetilde{H}}, w^{\prime}\right]\right) \geq \int_{0}^{1}-\max \widetilde{H}_{t} d t=-E^{+}(\widetilde{H}) .
$$

Next let us concatenate the two equations (4.14) for $\chi_{+}$as in (2.5) and $\chi_{-}=1-\chi_{+}$by considering one-parameter family of elongation function of the type

$$
\chi_{K}= \begin{cases}\chi_{+}(\cdot+K) & \text { for } \tau \leq-K+1, \\ \chi_{-}(\cdot-K+1) & \text { for } \tau \geq K-1, \\ 1 & \text { for }|\tau| \leq K-1\end{cases}
$$

for $1 \leq K \leq \infty$ and further deforming $\chi_{K=1}$ further down to $\chi_{K=0} \equiv 0$.

Proposition 5.4. Let $u$ be a finite energy solution for (4.14) of the elongation $\chi_{K}$ with asymptotic condition

$$
u(-\infty)=\ell_{p}, u(\infty)=\ell_{q}, w_{-} \# u \sim w_{+} .
$$

Then, we have

$$
\begin{aligned}
\mathcal{A}_{\ell_{a}}\left(\left[\ell_{q}, w_{+}\right]\right)-\mathcal{A}_{\ell_{a}}\left(\left[\ell_{p}, w_{-}\right]\right) & \geq-\left(E^{-}(\widetilde{H})+E^{+}(\widetilde{H})\right)=-\|\widetilde{H}\| \\
E_{\left(J, \widetilde{H} ; \chi_{K}\right)}(u) & \leq \int u^{*} \omega+\|\widetilde{H}\| .
\end{aligned}
$$


As we explained in Remark 4.3, we choose $\ell_{a}$ such that

$$
c\left(\widetilde{H} ; \ell_{a}\right)=\int_{0}^{1} \widetilde{H}\left(t, \ell_{a}(t)\right) d t=0 .
$$

Recall that $\ell_{a}^{\prime}=\mathfrak{g}_{H^{(0)}, H^{(1)}}^{-}\left(\ell_{a}\right)=\psi^{t} \circ \ell_{a}(t)$. For $v:[0,1] \times[0,1] \rightarrow X$, we set

$$
\left(\Phi_{H^{(0)}, H^{(1)}} v\right)(s, t)=\left(\psi^{t}\right)^{-1}(v(s, t)) .
$$

Since $\widetilde{H}$ is defined by (4.8), we find that

$$
E^{-}(\widetilde{H}) \leq E^{-}\left(H^{(0)}\right)+E^{+}\left(H^{(1)}\right), \quad E^{+}(\widetilde{H}) \leq E^{+}\left(H^{(0)}\right)+E^{-}\left(H^{(1)}\right) .
$$

Therefore the following propositions follow from Corollary 5.3 and Proposition 5.4, respectively.

Proposition 5.5. Let $\left(L^{(0)}, L^{(1)}\right)$ be a pair of compact Lagrangian submanifolds and $\left(L^{(0) \prime}, L^{(1) \prime}\right)$ another pair with

$$
L^{(0) \prime}=\phi_{H^{(0)}}^{1}\left(L^{(0)}\right), \quad L^{(1) \prime}=\phi_{H^{(1)}}^{1}\left(L^{(1)}\right)
$$

and let $H^{(0)}, H^{(1)}$ be the normalized Hamiltonians generating $\phi_{H^{(0)}}^{1}$ and $\phi_{H^{(1)}}^{1}$, respectively. Consider a pair $\left[\ell_{p}, w\right] \in \widetilde{\Omega}\left(L^{(0)}, L^{(1)} ; \ell_{a}\right)$ and $\left[\ell_{q^{\prime}}, w^{\prime}\right] \in$ $\widetilde{\Omega}\left(L^{(0) \prime}, L^{(1) \prime} ; \ell_{a}^{\prime}\right)$ for which there exists a solution $u$ of $(4.14)$ with $\chi=\chi_{+}$ as in (2.4) such that

$$
\lim _{\tau \rightarrow-\infty} u(\tau, \cdot)=\ell_{p}, \lim _{\tau \rightarrow+\infty} u(\tau, \cdot)=\mathfrak{g}_{H^{(0)}, H^{(1)}}^{+}\left(\ell_{q^{\prime}}\right), \Phi_{H^{(0)}, H^{(1)}}(w \# u) \sim w^{\prime} .
$$

Then, we have

$$
\mathcal{A}_{\ell_{a}^{\prime}}\left(\left[\ell_{q^{\prime}}, w^{\prime}\right]\right)-\mathcal{A}_{\ell_{a}}\left(\left[\ell_{p}, w\right]\right) \geq-\left(E^{-}\left(H^{(0)}\right)+E^{+}\left(H^{(1)}\right)\right) .
$$

Similarly, let $\left[\ell_{p^{\prime}}, w^{\prime}\right] \in \widetilde{\Omega}\left(L^{(0) \prime}, L^{(1) \prime} ; \ell_{a}^{\prime}\right)$ and $\left[\ell_{q}, w\right] \in \widetilde{\Omega}\left(L^{(0)}, L^{(1)} ; \ell_{a}\right)$. If there exists a solution $u$ of (4.14) with $\chi=\chi_{-}=1-\chi_{+}$, such that

$$
\lim _{\tau \rightarrow-\infty} u(\tau, \cdot)=\mathfrak{g}_{H^{(0)}, H^{(1)}}^{+}\left(\ell_{p^{\prime}}\right), \lim _{\tau \rightarrow+\infty} u(\tau, \cdot)=\ell_{q}, \Phi_{H^{(0)}, H^{(1)}}^{-1}\left(w^{\prime}\right) \# u \sim w,
$$

we have

$$
\mathcal{A}_{\ell_{a}}\left(\left[\ell_{q}, w\right]\right)-\mathcal{A}_{\ell_{a}^{\prime}}\left(\left[\ell_{p^{\prime}}, w^{\prime}\right]\right) \geq-\left(E^{+}\left(H^{(0)}\right)+E^{-}\left(H^{(1)}\right)\right) .
$$

Proposition 5.6. Let $\left(L^{(0)}, L^{(1)}\right)$ be a pair of compact Lagrangian submanifolds. If there exists a solution $u$ of (4.14) with $\chi=\chi_{K}$ in (5.6) satisfying

$$
\lim _{\tau \rightarrow-\infty} u(\tau, \cdot)=\ell_{p}, \lim _{\tau \rightarrow+\infty} u(\tau, \cdot)=\ell_{q}, w_{-} \# u \sim w_{+},
$$

then we have

$$
\mathcal{A}_{\ell_{a}}\left(\left[\ell_{q}, w_{+}\right]\right)-\mathcal{A}_{\ell_{a}}\left(\left[\ell_{p}, w_{-}\right]\right) \geq-\left(\left\|H^{(0)}\right\|+\left\|H^{(1)}\right\|\right)
$$


and

$$
E_{\left(J, \widetilde{H}, \chi_{K}\right)}(u) \leq \int u^{*} \omega+\left\|H^{(0)}\right\|+\left\|H^{(1)}\right\|
$$

\section{Corrected proofs of Theorem $\mathrm{J}$ and Theorem 6.1.25 [3]}

To keep the statement of Theorem $\mathrm{J}[\mathbf{3}]$ as it is, we need to modify construction of the chain map used in the proof of Theorem 6.1.25 [3]. The chain map is constructed from the filtered $A_{\infty}$-bimodule homomorphism, which we explain in the Appendix. When $L^{(0)}$ and $L^{(1)}$ are unobstructed in the sense of $[\mathbf{3}]$, the filtered $A_{\infty}$-bimodule homomorphism induces a chain map between Floer complexes, see the end of Appendix. In this Section, we use Propositions 5.5 and 5.6 in Section 5 to study the change of filtrations under the chain map.

In the rest of the paper, we assume that a Lagrangian submanifold is closed and relatively spin and a pair of Lagrangian submanifolds is relatively spin (Definition $1.6[\mathbf{3}]$ ) unless otherwise noted. In this discussion we use the $\mathbb{C}$-coefficients as in $[\mathbf{4}, \mathbf{5}]$ but one can also use the $\mathbb{Q}$-coefficients as in $[\mathbf{3}]$. As we explained at the beginning of Section 5 , we assume that $L^{(0)}$ and $L^{(1)}$ are transversal.

We first recall the definition of the universal Novikov ring $\Lambda_{\text {nov }}$ used in $[\mathbf{3}]$. An element of $\Lambda_{\text {nov }}$ is a formal sum

$$
\sum_{i=1}^{\infty} a_{i} T^{\lambda_{i}} e^{\mu_{i}}
$$

with $a_{i} \in \mathbb{C}, \lambda_{i} \in \mathbb{R}, \mu_{i} \in \mathbb{Z}$ such that $\lambda_{i} \leq \lambda_{i+1}$ and $\lim _{i \rightarrow \infty} \lambda_{i}=\infty$, unless it is a finite sum. Here, $T$ and $e$ are formal parameters. We define a valuation $\mathfrak{v}_{T}: \Lambda_{\text {nov }} \rightarrow \mathbb{R}$ by

$$
\mathfrak{v}_{T}\left(\sum_{i=1}^{\infty} a_{i} T^{\lambda_{i}} e^{\mu_{i}}\right)=\lambda_{1} .
$$

This induces a natural $\mathbb{R}$-filtration on $\Lambda_{\text {nov }}$ that in turn induces a nonArchimedean topology thereon. Then we define $\Lambda_{0, \text { nov }}$ to be the subring of $\Lambda_{\text {nov }}$ consisting of $\sum a_{i} T^{\lambda_{i}} e^{\mu_{i}}$ with $\mathfrak{v}_{T}\left(\sum_{i=1}^{\infty} a_{i} T^{\lambda_{i}} e^{\mu_{i}}\right) \geq 0$ and $\Lambda_{0, \text { nov }}^{+}$by the subring with $\mathfrak{v}_{T}>0$.

We define $C\left(L^{(1)}, L^{(0)} ; \Lambda_{\text {nov }}\right)$ as the $\Lambda_{\text {nov }}$-module generated by Crit $\mathcal{A}_{\ell_{a}}$, $a \in \pi_{0}\left(\Omega\left(L^{(0)}, L^{(1)}\right)\right)$ modulo the equivalence relation $\sim$ given in $(4.4)$. The filtration $\left\{F^{\lambda}\right\}$ on $C\left(L^{(1)}, L^{(0)} ; \Lambda_{\text {nov }}\right)$ is given by the action functional $\mathcal{A}_{\ell_{a}}$. See p.127 in $[\mathbf{3}]$. We can regard $C\left(L^{(1)}, L^{(0)} ; \Lambda_{\text {nov }}\right)$ as a free $\Lambda_{\text {nov-module }}$ generated by $L^{(0)} \cap L^{(1)}$ provided $L^{(0)}$ and $L^{(1)}$ intersect transversally. In such a situation, we can identify $F^{0} C\left(L^{(1)}, L^{(0)} ; \Lambda_{\text {nov }}\right)$ and the free $\Lambda_{0 \text {,nov-module }}$ generated by $L^{(0)} \cap L^{(1)}$. We defined a filtered $A_{\infty}$-bimodule structure on $C\left(L^{(1)}, L^{(0)} ; \Lambda_{\text {nov }}\right.$ ) in Theorem 3.7.21 in [3] (see also Definition 3.7.41 in 
[3]). By extending the coefficient ring to $\Lambda_{\text {nov }}$, we also have a filtered $A_{\infty^{-}}$ bimodule structure on $C\left(L^{(1)}, L^{(0)} ; \Lambda_{\text {nov }}\right)$. This construction does not rely on the choice of the base paths $\ell_{a}$. However, when we construct a weakly filtered $A_{\infty}$-bimodule homomorphism $C\left(L^{(1)}, L^{(0)} ; \Lambda_{\text {nov }}\right) \rightarrow C\left(L^{(1) \prime}, L^{(0) \prime} ; \Lambda_{\text {nov }}\right)$, we use the base paths $\ell_{a}$ and $\ell_{a}^{\prime}$. As we will see, the improved estimate in Section 5 is used to control the filtration change under the weakly filtered $A_{\infty}$-bimodule homomorphism. See the Appendix for the construction of the weakly filtered $A_{\infty}$-bimodule homomorphism using solutions of the CauchyRiemann equation perturbed by Hamiltonians.

6.1. Statement of Theorem $\mathbf{J}[\mathbf{3}]$. In [3], we associate a set $\mathcal{M}_{\text {weak,def }}(L)$ for each relatively spin Lagrangian submanifold $L$ of $(X, \omega)$ and the maps

$$
\pi_{\text {amb }}: \mathcal{M}_{\text {weak,def }}(L) \rightarrow H^{2}\left(X ; \Lambda_{0, \text { nov }}^{+}\right), \quad \mathfrak{P O}: \mathcal{M}_{\text {weak,def }}(L) \rightarrow \Lambda_{0, \text { nov }}^{+}
$$

such that the Floer cohomology $\operatorname{HF}\left(\left(L, \mathbf{b}_{1}\right),\left(L, \mathbf{b}_{0}\right) ; \Lambda_{0, \text { nov }}\right)$ can be defined whenever the following condition holds: $\mathcal{M}_{\text {weak,def }}(L) \neq \emptyset$ and

$$
\pi_{\mathrm{amb}}\left(\mathbf{b}_{1}\right)=\pi_{\mathrm{amb}}\left(\mathbf{b}_{0}\right), \quad \mathfrak{P O}\left(\mathbf{b}_{1}\right)=\mathfrak{P O}\left(\mathbf{b}_{0}\right) .
$$

See Theorem B [3]. When this condition is satisfied, we say $L$ is weakly unobstructed after bulk deformation. We set

$$
\mathcal{M}_{\text {weak }}(L)=\pi_{\text {amb }}^{-1}(0), \quad \mathcal{M}(L)=\pi_{\text {amb }}^{-1}(0) \cap \mathfrak{P O}^{-1}(0),
$$

whose elements are called weak bounding cochain (weak Maurer-Cartan element), bounding cochain (Maurer-Cartan element), respectively. See Section 3.6, especially Definitions 3.6.4 and 3.6.29 [3] for the precise definitions of bounding cochain and weak bounding cochain. More generally, for a relative spin pair $\left(L^{(1)}, L^{(0)}\right)$ of Lagrangian submanifolds and

$$
\begin{aligned}
\left(\mathbf{b}_{1}, \mathbf{b}_{0}\right) & \in\left\{\left(\mathbf{b}_{1}, \mathbf{b}_{0}\right) \mid \pi_{\mathrm{amb}}\left(\mathbf{b}_{1}\right)=\pi_{\mathrm{amb}}\left(\mathbf{b}_{0}\right), \mathfrak{P O}\left(\mathbf{b}_{1}\right)=\mathfrak{P O}\left(\mathbf{b}_{0}\right)\right\} \\
& =: \mathcal{M}_{\text {weak,def }}\left(L^{(1)}\right) \times_{\left(\pi_{\mathrm{amb}}, \mathfrak{P O}\right)} \mathcal{M}_{\text {weak,def }}\left(L^{(0)}\right),
\end{aligned}
$$

we can define the Floer cohomology $\operatorname{HF}\left(\left(L^{(1)}, \mathbf{b}_{1}\right),\left(L^{(0)}, \mathbf{b}_{0}\right) ; \Lambda_{0, \text { nov }}\right)$ over $\Lambda_{0, \text { nov }}$. By Theorem 6.1.20 [3], it is isomorphic to

$$
\Lambda_{0, \mathrm{nov}}^{\oplus a} \oplus \bigoplus_{i=1}^{b}\left(\Lambda_{0, \mathrm{nov}} / T^{\lambda_{i}} \Lambda_{0, \mathrm{nov}}\right)
$$

for some non-negative integer $a$ and positive real numbers $\lambda_{i}(i=1, \ldots, b)$. We call $a$ the Betti number and $\lambda_{i}$ the torsion exponent of the Floer cohomology. We note that $\operatorname{HF}\left(\left(L^{(1)}, \mathbf{b}_{1}\right),\left(L^{(0)}, \mathbf{b}_{0}\right) ; \Lambda_{0, \text { nov }}\right)$ is not invariant under the Hamiltonian isotopy. However, it is proved in $[\mathbf{3}]$ (see Theorem G (G.4)) that the Floer cohomology

$$
H F\left(\left(L^{(1)}, \mathbf{b}_{1}\right),\left(L^{(0)}, \mathbf{b}_{0}\right) ; \Lambda_{\text {nov }}\right)
$$


with $\Lambda_{\text {nov }}$ its coefficients is invariant under the Hamiltonian isotopy and satisfies

$$
H F\left(\left(L^{(1)}, \mathbf{b}_{1}\right),\left(L^{(0)}, \mathbf{b}_{0}\right) ; \Lambda_{\text {nov }}\right) \cong \Lambda_{\text {nov }}^{\oplus a} .
$$

In particular, when $a \neq 0, L^{(0)}, L^{(1)}$ can not be displaced from each other. On the other hand, when $a=0$, there is no obvious obstruction to the displacement. In this case, the torsion part of $H F\left(\left(L^{(1)}, \mathbf{b}_{1}\right),\left(L^{(0)}, \mathbf{b}_{0}\right) ; \Lambda_{0, \text { nov }}\right)$ provides some information on the Hofer distance and the displacement energy of the two.

Now, under the above brief review of Lagrangian Floer theory for a weakly unobstructed Lagrangian submanifold after bulk deformation, we can state Theorem $\mathrm{J}$ of $[\mathbf{3}]$.

Theorem 6.1 (Theorem $\mathbf{J}[3]$ ). Let $\left(L^{(0)}, L^{(1)}\right)$ be a relatively spin pair of Lagrangian submanifolds of $X$ and $L^{(1)}, L^{(0)}$ weakly unobstructed after bulk deformations. Let $\left(\mathbf{b}_{1}, \mathbf{b}_{0}\right) \in \mathcal{M}_{\text {weak,def }}\left(L^{(1)}\right) \times_{\pi_{\text {amb }}, \mathfrak{P O}} \mathcal{M}_{\text {weak,def }}\left(L^{(0)}\right)$ as in (6.1) and $\psi: X \rightarrow X$ a Hamiltonian diffeomorphism. Assume that $\psi\left(L^{(1)}\right)$ is transversal to $L^{(0)}$ and denote

$$
b(\|\psi\|)=\#\left\{i \mid \lambda_{i} \geq\|\psi\|\right\},
$$

where $\lambda_{i}$ are the torsion exponents as in (6.2) and $\|\phi\|$ is the Hofer norm defined by (2.2). Then we have

$$
\#\left(\psi\left(L^{(1)}\right) \cap L^{(0)}\right) \geq a+2 b(\|\psi\|) .
$$

Theorem 6.1 follows from the following Theorem 6.1.25 of [3] (see Section $6.5 .3[\mathbf{3}])$. The proof of Theorem 6.1.25 contained an error that we now fix.

We recall that a symplectic diffeomorphism $\psi:(X, L) \rightarrow\left(X, L^{\prime}\right)$ induces a bijection

$$
\psi_{*}: \mathcal{M}_{\text {weak,def }}(L) \rightarrow \mathcal{M}_{\text {weak,def }}\left(L^{\prime}\right)
$$

which is compatible with the maps $\pi_{\text {amb }}$ and $\mathfrak{P O}$. See Theorem B (B.3) [3].

Theorem 6.2 (Theorem 6.1.25 [3]). Let $\left(L^{(0)}, L^{(1)}\right)$ and $\left(\mathbf{b}_{1}, \mathbf{b}_{0}\right)$ be as in Theorem 6.1, and $\psi^{(i)}: X \rightarrow X(i=0,1)$ Hamiltonian diffeomorphisms. Put $L^{(i) \prime}=\psi^{(i)}\left(L^{(i)}\right)$. Let $\lambda_{\downarrow, i}, i=1, \ldots, b$ and $\lambda_{\downarrow, i}^{\prime} i=1, \ldots, b^{\prime}$ be the torsion exponents of the Floer cohomology

$H F\left(\left(L^{(1)}, \mathbf{b}_{1}\right),\left(L^{(0)}, \mathbf{b}_{0}\right) ; \Lambda_{0, \text { nov }}\right), H F\left(\left(L^{(1) \prime}, \psi_{*}^{(1)} \mathbf{b}_{1}\right),\left(L^{(0) \prime}, \psi_{*}^{(0)} \mathbf{b}_{0}\right) ; \Lambda_{0, \text { nov }}\right)$ respectively. We order them so that $\lambda_{\downarrow, i} \geq \lambda_{\downarrow, i+1}$ and $\lambda_{\downarrow, i}^{\prime} \geq \lambda_{\downarrow, i+1}^{\prime}$. Denote

$$
\nu_{0}=\operatorname{dist}\left(L^{(0)}, L^{(0) \prime}\right)+\operatorname{dist}\left(L^{(1)}, L^{(1) \prime}\right) .
$$


Then if $\lambda_{\downarrow, i}>\nu_{0}$, then $i \leq b^{\prime}$. Moreover, if $\lambda_{\downarrow, i}>\nu_{0}$ and $\lambda_{\downarrow, i}^{\prime}>\nu_{0}$, then we have

$$
\left|\lambda_{\downarrow, i}-\lambda_{\downarrow, i}^{\prime}\right| \leq \nu_{0} .
$$

In particular, $\lambda_{\downarrow, i}$ is continuous for each $i$ as long as $\lambda_{\downarrow, i}>0$.

Remark 6.3. Let $\lambda \in \mathbb{R}$ such that $\lambda>2\|H\|$. In the statement (6.5.30) in p. $391[\mathbf{3}]$, we obtained the chain maps

$$
\begin{aligned}
& \left.\left.\phi: T^{\lambda} C\left(L^{(1)}, L^{(0)} ; \Lambda_{0, \text { nov }}\right) \rightarrow T^{\lambda-\|H\|} C\left(L^{(1) \prime}, L^{(0) \prime}\right) ; \Lambda_{0, \text { nov }}\right)\right) \\
& \phi^{\prime}: T^{\lambda-\|H\|} C\left(L^{(1)}, L^{(0)} ; \Lambda_{0, \text { nov }}\right) \rightarrow T^{\lambda-2\|H\|} C\left(L^{(1)}, L^{(0)} ; \Lambda_{0, \text { nov }}\right) .
\end{aligned}
$$

The above-mentioned error lies in the fact that the composition of (6.7) and (6.8) is only chain homotopy equivalent to the inclusion

$$
\mathfrak{i}: T^{\lambda} C\left(L^{(1)}, L^{(0)} ; \Lambda_{0, \text { nov }}\right) \longrightarrow T^{\lambda-2\|H\|} C\left(L^{(1)}, L^{(0)} ; \Lambda_{0, \text { nov }}\right)
$$

if we use the original energy estimate given in Proposition 5.3.20 (Proposition 5.3.45) [3]. Therefore, we need to replace the rest of the proof by the following argument, which uses the construction of an optimal chain maps combining the intertwining transformations explained in the previous sections and the improved energy estimate.

6.2. Proof of Theorem 6.2. In this subsection, we prove Theorem 6.2.

Consider the pair $\left(\psi^{(0)}, \psi^{(1)}\right)$ of Hamiltonian diffeomorphisms. As in [3], to simplify the notation, we restrict ourselves to the case of a transverse pair $\left(L^{(0)}, L^{(1)}\right)$ where both $L^{(i)}$ are unobstructed, i.e., $\mathcal{M}\left(L^{(i)}\right) \neq \emptyset$. Then using bounding cochains $b_{i} \in \mathcal{M}\left(L^{(i)}\right)$, we can define the coboundary operator $\delta_{b_{1}, b_{0}}$ on the filtered $A_{\infty}$ bimodule $C\left(L^{(1)}, L^{(0)} ; \Lambda_{0, \text { nov }}\right)=$ $F^{0} C\left(L^{(1)}, L^{(0)} ; \Lambda_{\text {nov }}\right)$. (See Section 3.7.4 [3].) Similarly, we have the coboundary operator $\delta_{b_{1}^{\prime}, b_{0}^{\prime}}$ on $C\left(L^{(1) \prime}, L^{(0) \prime} ; \Lambda_{0, \text { nov }}\right)=F^{0} C\left(L^{(1) \prime}, L^{(0) \prime} ; \Lambda_{\text {nov }}\right)$, where we put $b_{i}^{\prime}:=\psi_{*}^{(i)} b_{i}$.

Let $\delta>0$ be given. We consider any Hamiltonian isotopy $\phi_{H^{(0)}}, \phi_{H^{(1)}}$ generated by $H^{(0)}, H^{(1)}$ respectively such that $\phi_{H^{(i)}}^{1}=\psi^{(i)}$,

$$
L^{(0) \prime}=\psi^{(0)}\left(L^{(0)}\right), \quad L^{(1) \prime}=\psi^{(1)}\left(L^{(1)}\right)
$$

and

$$
\operatorname{leng}\left(\phi_{H^{(0)}}\right)+\operatorname{leng}\left(\phi_{H^{(1)}}\right) \leq \operatorname{dist}\left(L^{(0)}, L^{(0) \prime}\right)+\operatorname{dist}\left(L^{(1)}, L^{(1) \prime}\right)+\delta .
$$

Denote

$$
\nu_{-}=E^{-}\left(H^{(0)}\right)+E^{+}\left(H^{(1)}\right), \quad \nu_{+}=E^{+}\left(H^{(0)}\right)+E^{-}\left(H^{(1)}\right)
$$

and

$$
\nu:=\nu_{-}+\nu_{+}=\left\|H^{(0)}\right\|+\left\|H^{(1)}\right\|=\operatorname{leng}\left(\phi_{H^{(0)}}\right)+\operatorname{leng}\left(\phi_{H^{(1)}}\right) .
$$


We note that we can make $\nu$ as close to $\nu_{0}$ in (6.5) as we want. See Remark 6.5.

We construct a weakly filtered $A_{\infty}$ bimodule homomorphism

$$
\phi: C\left(L^{(1)}, L^{(0)} ; \Lambda_{\text {nov }}\right) \rightarrow C\left(L^{(1) \prime}, L^{(0) \prime} ; \Lambda_{\text {nov }}\right) .
$$

One such construction is provided in [3]. See (6.5.14) and (6.5.15) therein.

However, we would like to have an additional property that is required in Theorem 6.2 above. In [3], we used the moduli spaces of solutions for (4.19), which is the equation of moving Lagrangian boundary value problem. In this paper, we use the moduli spaces of solutions $u$ for (4.14), instead of (4.19), with $\chi=\chi_{+}$such that

$$
\lim _{\tau \rightarrow-\infty} u(\tau, \cdot)=\ell_{p}, \lim _{\tau \rightarrow+\infty} u(\tau, \cdot)=\mathfrak{g}_{H^{(0)}, H^{(1)}}\left(\ell_{q^{\prime}}\right), \Phi_{H^{(0)}, H^{(1)}}(w \# u) \sim w^{\prime}
$$

as in Proposition 5.5. See Appendix. Then by identifying Crit $\mathcal{A}_{\widetilde{H}, \ell_{a}}$ with Crit $\mathcal{A}_{\ell_{a}^{\prime}}$ we obtain a weakly filtered $A_{\infty}$ bimodule homomorphism $\phi$ in a way similar to Lemma 5.3.25 and Lemma 5.3.8 in [3]. The weakly filtered $A_{\infty}$ bimodule homomorphism induces a morphism of cochain complexes with differentials $\delta_{b_{0}, b_{1}}$, resp. $\delta_{b_{0}^{\prime}, b_{1}^{\prime}}$, which we also denote by $\phi$ by an abuse of notation:

$$
\phi: C\left(L^{(1)}, L^{(0)} ; \Lambda_{\text {nov }}\right) \rightarrow C\left(L^{(1) \prime}, L^{(0) \prime} ; \Lambda_{\text {nov }}\right) .
$$

Similarly, we use the moduli spaces of solutions $u$ for (4.14) with $\chi=$ $\chi_{-}$such that

$$
\lim _{\tau \rightarrow-\infty} u(\tau, \cdot)=\mathfrak{g}_{H^{(0)}, H^{(1)}}^{+}\left(\ell_{p^{\prime}}\right), \lim _{\tau \rightarrow+\infty} u(\tau, \cdot)=\ell_{q}, \Phi_{H^{(0)}, H^{(1)}}^{-1}\left(w^{\prime}\right) \# u \sim w,
$$

to obtain

$$
\phi^{\prime}: C\left(L^{(1) \prime}, L^{(0) \prime} ; \Lambda_{\text {nov }}\right) \rightarrow C\left(L^{(1)}, L^{(0)} ; \Lambda_{\text {nov }}\right) .
$$

Since we choose $\ell_{a}$ in such a way that $c\left(\widetilde{H}, \ell_{a}\right)=0$ for all $a \in$ $\pi_{0}\left(\Omega\left(L^{(0)}, L^{(1)}\right)\right)$, Proposition 5.5 implies that these composition satisfies

$$
\phi: F^{\lambda} C\left(L^{(1)}, L^{(0)} ; \Lambda_{\text {nov }}\right) \rightarrow F^{\lambda-\nu-} C\left(L^{(1) \prime}, L^{(0) \prime} ; \Lambda_{\text {nov }}\right) .
$$

Similarly, we obtain

$$
\phi^{\prime}: F^{\lambda} C\left(L^{(1) \prime}, L^{(0) \prime} ; \Lambda_{\text {nov }}\right) \rightarrow F^{\lambda-\nu_{+}} C\left(L^{(1)}, L^{(0)} ; \Lambda_{\text {nov }}\right) .
$$

This leads to the chain map

$$
\phi^{\prime} \circ \phi: F^{\lambda} C\left(L^{(1)}, L^{(0)} ; \Lambda_{\text {nov }}\right) \longrightarrow F^{\lambda-\nu} C\left(L^{(1)}, L^{(0)} ; \Lambda_{\text {nov }}\right) .
$$

Equivalently, we can rewrite these into the chain maps

$$
T^{\nu_{-}} \phi: F^{\lambda} C\left(L^{(1)}, L^{(0)} ; \Lambda_{\text {nov }}\right) \rightarrow F^{\lambda} C\left(L^{(1) \prime}, L^{(0) \prime} ; \Lambda_{\text {nov }}\right)
$$

and

$$
T^{\nu_{+}} \phi^{\prime}: F^{\lambda} C\left(L^{(1) \prime}, L^{(0) \prime} ; \Lambda_{\text {nov }}\right) \rightarrow F^{\lambda} C\left(L^{(1)}, L^{(0)} ; \Lambda_{\text {nov }}\right) .
$$


Setting $\lambda=0$, we have

$$
\begin{gathered}
\left(T^{\nu_{-}} \phi\right)_{*}: H F\left(\left(L^{(1)}, b_{1}\right),\left(L^{(0)}, b_{0}\right) ; \Lambda_{0, \text { nov }}\right) \\
\rightarrow H F\left(\left(L^{(1) \prime}, b_{1}^{\prime}\right),\left(L^{(0) \prime}, b_{0}^{\prime}\right) ; \Lambda_{0, \text { nov }}\right)
\end{gathered}
$$

and

$$
\begin{aligned}
& \left(T^{\nu_{+}} \phi^{\prime}\right)_{*}: H F\left(\left(L^{(1) \prime}, b_{1}^{\prime}\right),\left(L^{(0) \prime}, b_{0}^{\prime}\right) ; \Lambda_{0, \text { nov }}\right) \\
& \quad \rightarrow H F\left(\left(L^{(1)}, b_{1}\right),\left(L^{(0)}, b_{0}\right) ; \Lambda_{0, \text { nov }}\right)
\end{aligned}
$$

respectively. We denote

$$
\mathfrak{i}: F^{\lambda} C\left(L^{(1)}, L^{(0)} ; \Lambda_{\text {nov }}\right) \hookrightarrow F^{\lambda-\nu} C\left(L^{(1)}, L^{(0)} ; \Lambda_{\text {nov }}\right)
$$

the inclusion induced homomorphism.

Lemma 6.4. The two maps

$$
\left(T^{\nu_{+}} \phi^{\prime}\right) \circ\left(T^{\nu_{-}} \phi\right), T^{\nu_{i}}: F^{\lambda} C\left(L^{(1)}, L^{(0)} ; \Lambda_{\text {nov }}\right) \longrightarrow F^{\lambda} C\left(L^{(1)}, L^{(0)} ; \Lambda_{\text {nov }}\right)
$$

are chain homotopic to each other.

Proof. This statement follows from the arguments in p. 390-391 [3], and also from the explicit energy bound (5.12) in Proposition 5.6 for solutions $u$ of (4.14) with $\widetilde{H}$ and $\chi=\chi_{K}, 0 \leq K<\infty$, which are used to define the chain homotopy map. Recall that $\chi_{K}$ extends smoothly to $K=0$ as the constant function zero. Then the moduli spaces of solutions of (4.14) with $\chi=\chi_{K}$ in (5.6) defines a chain homotopy between $\phi^{\prime} \circ \phi$ and the identity.

As for the filtrations, we apply (5.11) in Proposition 5.6 to all the elements in the associated parameterized moduli space defining the chain homotopy and find that the energy loss is bounded by $\nu$ for all $K$. This proves that $\phi^{\prime} \circ \phi$ is chain homotopic to $\mathfrak{i}$ as a map

$$
F^{\lambda} C\left(L^{(1)}, L^{(0)} ; \Lambda_{\text {nov }}\right) \rightarrow F^{\lambda-\nu} C\left(L^{(1)}, L^{(0)} ; \Lambda_{\text {nov }}\right) .
$$

From now on, we consider the case that $\lambda=0$. Then, we have

$$
\left(T^{\nu_{+}} \phi^{\prime}\right)_{*} \circ\left(T^{\nu_{-}} \phi\right)_{*}=T^{\nu}
$$

where

$$
T^{\nu}: H F\left(\left(L^{(1)}, b_{1}\right),\left(L^{(0)}, b_{0}\right) ; \Lambda_{0, \text { nov }}\right) \rightarrow H F\left(\left(L^{(1)}, b_{1}\right),\left(L^{(0)}, b_{0}\right) ; \Lambda_{0, \text { nov }}\right)
$$

is the map $x \mapsto T^{\nu} x$.

Since

and

$$
\begin{aligned}
\left(T^{\nu_{-}} \phi\right)_{*} & : H F\left(\left(L^{(1)}, b_{1}\right),\left(L^{(0)}, b_{0}\right) ; \Lambda_{0, \text { nov }}\right) \\
& \rightarrow H F\left(\left(L^{(1) \prime}, b_{1}^{\prime}\right),\left(L^{(0) \prime}, b_{0}^{\prime}\right) ; \Lambda_{0, \text { nov }}\right)
\end{aligned}
$$

$$
\begin{aligned}
\left(T^{\nu+} \phi^{\prime}\right)_{*}: & H F\left(\left(L^{(1) \prime}, b_{1}^{\prime}\right),\left(L^{(0) \prime}, b_{0}^{\prime}\right) ; \Lambda_{0, \text { nov }}\right) \\
& \rightarrow H F\left(\left(L^{(1)}, b_{1}\right),\left(L^{(0)}, b_{0}\right) ; \Lambda_{0, \text { nov }}\right)
\end{aligned}
$$


are $\Lambda_{0, \text { nov }}$-module homomorphisms, we have, for any $\lambda>0$,

$$
\begin{aligned}
\left(T^{\nu_{-}} \phi\right)_{*}: & T^{\lambda} H F\left(\left(L^{(1)} b_{1}\right),\left(L^{(0)}, b_{0}\right) ; \Lambda_{0, \text { nov }}\right) \\
& \rightarrow T^{\lambda} H F\left(\left(L^{(1) \prime}, b_{1}^{\prime}\right),\left(L^{(0) \prime}, b_{0}^{\prime}\right) ; \Lambda_{0, \text { nov }}\right)
\end{aligned}
$$

and

$$
\begin{aligned}
\left(T^{\nu_{+}} \phi^{\prime}\right)_{*}: & T^{\lambda} H F\left(\left(L^{(1) \prime}, b_{1}^{\prime}\right),\left(L^{(0) \prime}, b_{0}^{\prime}\right) ; \Lambda_{0, \text { nov }}\right) \\
& \rightarrow T^{\lambda} H F\left(\left(L^{(1)} b_{1}\right),\left(L^{(0)}, b_{0}\right) ; \Lambda_{0, \text { nov }}\right) .
\end{aligned}
$$

Since $\left(T^{\nu_{+}} \phi^{\prime}\right)_{*} \circ\left(T^{\nu-} \phi\right)_{*}$ is equal to the multiplication by $T^{\nu}$, the minimal number of generators of $\operatorname{Im}\left(T^{\nu_{+}} \phi^{\prime}\right)_{*} \circ\left(T^{\nu_{-}} \phi\right)_{*}$ is equal to $a+b(\lambda+\nu)$ if $\lambda+\nu \notin\left\{\lambda_{\downarrow} \mid i=1, \ldots, b\right\}$. On the other hand, the minimal number of generators of $T^{\lambda} H F\left(\left(L^{(1) \prime}, b_{1}^{\prime}\right),\left(L^{(0) \prime}, b_{0}^{\prime}\right) ; \Lambda_{0, \text { nov }}\right)$ is equal to $a+b^{\prime}(\lambda)$ if $\lambda \notin\left\{\lambda_{\downarrow, i}^{\prime} \mid i=1, \ldots, b^{\prime}\right\}$. Here

$$
b^{\prime}(\lambda)=\#\left\{i \mid \lambda_{\downarrow, i}^{\prime} \geq \lambda\right\} .
$$

Therefore we have

$$
a+b(\lambda+\nu) \leq a+b^{\prime}(\lambda)
$$

for $\lambda \notin\left\{\lambda_{\downarrow, i}-\nu \mid i=1, \ldots, b\right\} \cup\left\{\lambda_{\downarrow, i}^{\prime} \mid i=1, \ldots, b^{\prime}\right\}$ cf. Lemma 6.5.31 in [3]. This implies that $\lambda_{\downarrow, i}^{\prime} \geq \lambda_{\downarrow, i}-\nu$, whenever $\lambda_{\downarrow, i}>\nu$.

Since this holds for all Hamiltonian isotopies $\phi_{H^{(0)}}, \phi_{H^{(1)}}$ satisfying (6.9), (6.10) and for any $\delta>0$, we obtain

$$
\text { if } \lambda_{\downarrow, i}>\nu, \quad \lambda_{\downarrow, i} \leq \nu+\lambda_{\downarrow, i}^{\prime} .
$$

By changing the role of $L^{(1)}, L^{(0)}$ with $L^{(1) \prime}, L^{(0) \prime}$ we also obtain

$$
\text { if } \lambda_{\downarrow, i}^{\prime}>\nu, \quad \lambda_{\downarrow, i}^{\prime} \leq \nu+\lambda_{\downarrow, i} .
$$

Theorem 6.2 follows.

Remark 6.5. With given fixed $L^{(0) \prime} \in \mathfrak{I s o}\left(L^{(0)}\right)$ and $L^{(1) \prime} \in \mathfrak{I s o}\left(L^{(1)}\right)$, we may consider all possible Hamiltonian isotopy with given end points and take the infimum of leng $\left(\phi_{H^{(0)}}\right)+\operatorname{leng}\left(\phi_{H^{(1)}}\right)$ over all $H^{(0)}$ and $H^{(1)}$ such that

$$
\phi_{H^{(0)}}^{1}\left(L^{(0)}\right)=L^{(0) \prime}, \quad \phi_{H^{(1)}}^{1}\left(L^{(1)}\right)=L^{(1) \prime} .
$$

In this way, we can make $\operatorname{leng}\left(\phi_{H^{(0)}}\right)+\operatorname{leng}\left(\phi_{H^{(1)}}\right)$ as close to the sum

$$
\operatorname{dist}\left(L^{(0)}, L^{(0) \prime}\right)+\operatorname{dist}\left(L^{(1)}, L^{(1) \prime}\right)
$$

as we want. 


\section{Torsion threshold and displacement energy}

7.1. Torsion threshold. As we mentioned, the torsion exponents of the Floer cohomology have some information on the displacement energy of Lagrangian submanifolds. We introduce the following notion to describe a relation between the torsion exponents and the displacement energy.

Definition 7.1. Let $L^{(1)}, L^{(0)}$ be weakly unobstructed Lagrangian submanifolds after bulk deformations. Let

$$
\left(\mathbf{b}_{1}, \mathbf{b}_{0}\right) \in \mathcal{M}_{\text {weak,def }}\left(L^{(1)}\right) \times_{\left(\pi_{\text {amb }}, \mathfrak{P O}\right)} \mathcal{M}_{\text {weak,def }}\left(L^{(0)}\right)
$$

as in (6.1). Suppose $\operatorname{HF}\left(\left(L^{(1)}, \mathbf{b}_{1}\right),\left(L^{(0)}, \mathbf{b}_{0}\right) ; \Lambda_{\text {nov }}\right)=0$. We denote by $\lambda_{i}$ its torsion exponents defined by (6.2).

(1) We define

$$
\mathfrak{T}\left(\left(L^{(1)}, \mathbf{b}_{1}\right),\left(L^{(0)}, \mathbf{b}_{0}\right)\right)=\max _{i} \lambda_{i}
$$

and call it the torsion threshold of the pair $\left(L^{(0)}, L^{(1)}\right)$ relative to $\left(\mathbf{b}_{1}, \mathbf{b}_{0}\right)$.

(2) We define

$$
\mathfrak{T}\left(L^{(1)}, L^{(0)}\right)=\sup _{\left(\mathbf{b}_{1}, \mathbf{b}_{0}\right)} \mathfrak{T}\left(\left(L^{(1)}, \mathbf{b}_{1}\right),\left(L^{(0)}, \mathbf{b}_{0}\right)\right)
$$

and call it the torsion threshold of the pair $\left(L^{(0)}, L^{(1)}\right)$.

When $\operatorname{HF}\left(\left(L^{(1)}, \mathbf{b}_{1}\right),\left(L^{(0)}, \mathbf{b}_{0}\right) ; \Lambda_{\text {nov }}\right) \neq 0$ for some $\left(\mathbf{b}_{1}, \mathbf{b}_{0}\right)$, we define

$$
\mathfrak{T}\left(L^{(1)}, L^{(0)}\right)=\infty \text {. }
$$

(3) We just denote $\mathfrak{T}((L, \mathbf{b}),(L, \mathbf{b}))$ and $\mathfrak{T}(L, L)$ by $\mathfrak{T}(L, \mathbf{b})$ and $\mathfrak{T}(L)$ respectively.

We now specialize the energy estimate in the previous section to the particular case

$$
\left(L^{(0)}, L^{(1)}\right)=(L, L), \quad\left(L^{(0) \prime}, L^{(1) \prime}\right)=\left(L, \psi^{(1)}(L)\right)
$$

with the displacing condition

$$
L \cap \psi^{(1)}(L)=\emptyset .
$$

Then the following theorem relating the displacement energy and the torsion threshold of $L$ is a special case of Theorem J. For readers' convenience, we give its proof that specializes the proof of Theorem $\mathrm{J}$ to this particular context.

Theorem 7.2. Let $L$ be a relatively spin closed Lagrangian submanifold of $(X, \omega)$. Suppose that $L$ is weakly unobstructed after bulk deformation and displaceable. We denote by $e(L)\left(=e^{X}(L)\right)$ its displacement energy. Let the torsion threshold of $\operatorname{HF}\left((L, \mathbf{b}),(L, \mathbf{b}) ; \Lambda_{0, \text { nov }}\right)$ be positive, i.e., assume 
$\mathfrak{T}(L, \mathbf{b})>0$. Then, we have

$$
e(L) \geq \mathfrak{T}(L, \mathbf{b})
$$

for any $\mathbf{b} \in \mathcal{M}_{\text {weak,def }}(L)$. In particular, we have $e(L) \geq \mathfrak{T}(L)$.

Proof. Suppose to the contrary that there exists a sufficiently small $\delta>0$ and an element $\mathfrak{b} \in \mathcal{M}_{\text {weak,def }}(L)$ such that

$$
e(L)<\mathfrak{T}(L, \mathbf{b})-\delta .
$$

Pick a Hamiltonian $H$ and its associated Hamiltonian isotopy $\phi_{H}$ such that

$$
\phi_{H}^{1}(L) \cap L=\emptyset, \quad\|H\| \leq e(L)+\delta .
$$

In particular, we also have

$$
\|H\|<\mathfrak{T}(L, \mathbf{b}) .
$$

Now we recall from (6.13) that $\phi_{*}^{\prime} \circ \phi_{*}$ restricts to

$$
T^{\lambda} H F\left((L, \mathbf{b}),(L, \mathbf{b}) ; \Lambda_{0, \text { nov }}\right) \rightarrow T^{\lambda-\|H\|} H F\left((L, \mathbf{b}),(L, \mathbf{b}) ; \Lambda_{0, \text { nov }}\right) .
$$

and satisfies

as a map

$$
T^{\lambda} H F\left((L, \mathbf{b}),(L, \mathbf{b}) ; \Lambda_{0, \text { nov }}\right) \rightarrow T^{\lambda-\|H\|} H F\left((L, \mathbf{b}),(L, \mathbf{b}) ; \Lambda_{0, \text { nov }}\right)
$$

for all $\lambda \in \mathbb{R}$.

We now specialize to the case $\lambda=\|H\|$. In this case,

$$
T^{\lambda-\|H\|} H F\left((L, \mathbf{b}),(L, \mathbf{b}) ; \Lambda_{0, \text { nov }}\right)=H F\left((L, \mathbf{b}),(L, \mathbf{b}) ; \Lambda_{0, \text { nov }}\right) .
$$

Since $\lambda<\mathfrak{T}(L, \mathbf{b})$, the image of the inclusion-induced map

$$
\mathfrak{i}_{*}: T^{\lambda} H F\left((L, \mathbf{b}),(L, \mathbf{b}) ; \Lambda_{0, \text { nov }}\right) \rightarrow H F\left((L, \mathbf{b}),(L, \mathbf{b}) ; \Lambda_{0, \text { nov }}\right)
$$

is not trivial by the definition of $\mathfrak{T}(L, \mathbf{b})$.

On the other hand, $H F\left(\left(\phi_{H}^{1}(L), \phi_{H *}^{1} \mathbf{b}\right),(L, \mathbf{b}) ; \Lambda_{0, \text { nov }}\right)=\{0\}$ by the hypothesis $L \cap \phi_{H}^{1}(L)=\emptyset$ and hence $\phi_{*}=0=\phi_{*}^{\prime}$ which implies $\phi_{*}^{\prime} \circ \phi_{*}=0$.

Therefore, the equality $\phi_{*}^{\prime} \circ \phi_{*}=\left(\phi^{\prime} \circ \phi\right)_{*}=\mathfrak{i}_{*}$ with $\lambda=\|H\|$ in $(7.2)$ gives rise to a contradiction. This finishes the proof.

An interesting example to which Theorem 7.2 applies but the version without bulk deformations may not apply can be constructed using the idea of $[7]$.

7.2. $\mathfrak{P O}$-threshold for toric fibers. Let $(X, \omega)$ be a smooth compact toric manifold of $\operatorname{dim}_{\mathbb{C}} X=n$. Let $\pi: X \rightarrow P \subset \mathbb{R}^{n}$ be a moment map of $X$ and $L(u)=\pi^{-1}(u)$ for $u \in \operatorname{Int} P$ a Lagrangian torus fiber. In Definition 5.10 and Theorem $5.11[\mathbf{4}]$ we introduced the $\mathfrak{P O}$-threshold $\overline{\mathfrak{E}}(L(u))$ and proved an estimate of the displacement energy of $L(u)$ as

$$
e(L(u)) \geq 2 \pi \overline{\mathfrak{E}}(L(u)) .
$$


The constant $2 \pi$ appears in the right-hand side, because we used $T$ in $[4]$ as $T^{2 \pi}$ in $[\mathbf{3}]$ and this paper. See the paragraph around (4.3) in p.51 [4]. In this subsection, we generalize this inequality by involving bulk deformations. In [5] we developed bulk deformations of Lagrangian Floer theory of $L(u)$. We briefly recall the result of Section $3[\mathbf{5}]$ in which we defined Floer cohomology

$$
H F\left((L(u), \mathfrak{b}, b),(L(u), \mathfrak{b}, b) ; \Lambda_{0}\right)=\frac{\operatorname{Ker} \delta^{\mathfrak{b}, b}}{\operatorname{Im} \delta^{\mathfrak{b}, b}}
$$

for any $(\mathfrak{b}, b) \in \mathcal{A}\left(\Lambda_{0}\right) \times H^{1}\left(L(u) ; \Lambda_{0}\right) \cong\left(\Lambda_{0}\right)^{B} \times\left(\Lambda_{0}\right)^{n}$. Here $\mathcal{A}\left(\Lambda_{0}\right)=$ $\mathcal{A} \otimes_{\mathbb{Z}} \Lambda_{0}$ and $\mathcal{A}=\mathcal{A}(\mathbb{Z})$ is the free abelian group generated by the $T^{n_{-}}$ equivariant algebraic cycles of toric manifold $X$. By Theorem $3.14[\mathbf{5}]$ the potential function $\mathfrak{P O}$ can be regarded as a function on $\left(\Lambda_{0}\right)^{B} \times\left(\Lambda_{0}\right)^{n}$. The following is Theorem $3.16[\mathbf{5}]$.

Theorem 7.3. Let $(\mathfrak{b}, \mathfrak{x}) \in\left(\Lambda_{0}\right)^{B} \times\left(\Lambda_{0}\right)^{n}$. We assume

$$
\frac{\partial \mathfrak{P O}}{\partial x_{i}}(\mathfrak{b}, \mathfrak{x})=0
$$

for $i=1, \ldots, n$. Then we have

$$
H F\left(\left(L\left(u_{0}\right), \mathfrak{b}, \mathfrak{x}\right),\left(L\left(u_{0}\right), \mathfrak{b}, \mathfrak{x}\right) ; \Lambda_{0}\right) \cong H\left(T^{n} ; \Lambda_{0}\right) .
$$

If we assume

$$
\frac{\partial \mathfrak{P O}}{\partial x_{i}}(\mathfrak{b}, \mathfrak{x}) \equiv 0 \quad \bmod T^{\mathcal{N}}
$$

then we have

$$
H F\left(\left(L\left(u_{0}\right), \mathfrak{b}, \mathfrak{x}\right),\left(L\left(u_{0}\right), \mathfrak{b}, \mathfrak{x}\right) ; \Lambda_{0} / T^{\mathcal{N}}\right) \cong H\left(T^{n} ; \Lambda_{0} / T^{\mathcal{N}}\right) .
$$

We also recall the following definition (Definition $3.17[\mathbf{5}]$ ) that is a generalization of Definition 4.11 [4].

Definition 7.4. Let $L(u)$ be a Lagrangian fiber of a compact toric manifold $(X, \omega)$. We say that $L(u)$ is bulk-balanced if there exist sequences $\omega_{j}, P_{j}, u_{j}$, $\mathfrak{b}_{j}, \mathfrak{x}_{j}$, and $\mathcal{N}_{j}$ with the following properties.

(1) $\left(X, \omega_{j}\right)$ is a sequence of toric manifolds such that $\lim _{j \rightarrow \infty} \omega_{j}=\omega$.

(2) $P_{j}$ is a moment polytope of $\left(X, \omega_{j}\right)$. It converges to the moment polytope $P$ of $(X, \omega)$.

(3) $u_{j} \in P_{j}$ and $\lim _{j \rightarrow \infty} u_{j}=u$.

(4) $\mathfrak{b}_{j} \in \mathcal{A}\left(\Lambda_{+}(\mathbb{C})\right), \mathfrak{x}_{j} \in H^{1}\left(L\left(u_{i}\right) ; \Lambda_{0}(\mathbb{C})\right), \mathcal{N}_{j} \in \mathbb{R}_{+}$.

(5)

$$
H F\left(\left(L\left(u_{j}\right), \mathfrak{b}_{j}, \mathfrak{x}_{j}\right),\left(\left(L\left(u_{j}\right), \mathfrak{b}_{j}, \mathfrak{x}_{j}\right) ; \Lambda_{0}(\mathbb{C}) / T^{\mathcal{N}_{j}}\right) \cong H\left(T^{n} ; \Lambda_{0}(\mathbb{C}) / T^{\mathcal{N}_{j}}\right) .\right.
$$

(6) $\lim _{i \rightarrow \infty} \mathcal{N}_{j}=\infty$.

Now we define a bulk version of $\mathfrak{P O}$-threshold as follows. This is a straightforward generalization of $\overline{\mathfrak{E}}(L(u))$ in Definition $5.10[\mathbf{4}]$. 
Definition 7.5. We define the number $\mathfrak{E}^{\text {bulk }}(L(u))$ as the supremum of all $\lambda$ such that there exists $(\mathfrak{b}, \mathfrak{x}) \in\left(\Lambda_{0}\right)^{B} \times\left(\Lambda_{0}\right)^{n}$ satisfying

$$
\frac{\partial \mathfrak{P O}}{\partial x_{i}}(\mathfrak{b}, \mathfrak{x}) \equiv 0 \quad \bmod T^{\lambda},
$$

for $i=1, \ldots, n$. We put

$$
\overline{\mathfrak{E}}^{\mathrm{bulk}}(L(u))=\lim \sup \mathfrak{E}^{\mathrm{bulk}}\left(L\left(u_{j}\right)\right),
$$

where $\lim \sup$ is taken over all sequences $\omega_{j}, P_{j}, u_{j}$ as in Definition 7.4 (1)-(3). We call $\overline{\mathfrak{E}}^{\mathrm{bulk}}(L(u))$ the bulk $\mathfrak{P O}$-threshold of $L(u)$.

Remark 7.6. In Definition 5.10 [4], we defined two numbers $\overline{\mathfrak{E}}(L(u))$ and $\mathfrak{E}(L(u))$. Namely, the $\mathfrak{P O}$-threshold $\mathfrak{E}(L(u))$ is the supremum of all $\lambda$, such that there exists $\mathfrak{x} \in\left(\Lambda_{0}\right)^{n}$ satisfying

$$
\frac{\partial \mathfrak{P O}}{\partial x_{i}}(\mathfrak{x}) \equiv 0 \quad \bmod T^{\lambda}, \quad i=1, \ldots, n .
$$

We define $\overline{\mathfrak{E}}(L(u))$ in the same way as in the definition of $\mathfrak{E}^{\mathrm{bulk}}(L(u))$, Definition 7.5 but the supremum is taken with the restriction $\mathfrak{b}_{i}, \mathfrak{b}$ are all zero.

It is easy to see

$$
\mathfrak{E}(L(u)) \leq \overline{\mathfrak{E}}(L(u)) \leq \overline{\mathfrak{E}}^{\mathrm{bulk}}(L(u)) .
$$

The first equality in (7.7) does not hold in general. See Example 10.17 [4].

Then, we can show the following.

Theorem 7.7.

$$
e(L(u)) \geq 2 \pi \overline{\mathfrak{E}}^{\mathrm{bulk}}(L(u)) .
$$

Proof. Once we prove Theorem 7.3 which is the bulk deformation version of Theorem 4.10 [4], the proof goes in the same way as that of Theorem $5.11[4]$.

It would be interesting to see if the following holds:

Problem 7.8. Does the equality hold in (7.8)? What is the precise relationship between the two thresholds, the torsion threshold and the bulk $\mathfrak{P O}$-threshold for the toric fiber $L(u)$ in general?

\section{Displacement of polydisks inside cylinders in high dimensions}

In this section, we prove Theorem 1.3 and Theorem 1.4 stated in Section 1. We recall the polydisks in $\mathbb{C}^{n}$ denoted by

$$
D\left(a_{1}, a_{2}, \ldots, a_{n}\right)=\left\{\left.\left(z_{1}, \ldots, z_{n}\right) \in \mathbb{C}^{n}|\pi| z_{1}\right|^{2}<a_{1}, \ldots, \pi\left|z_{n}\right|^{2}<a_{n}\right\}
$$


where $a_{1} \leq a_{2} \leq \cdots \leq a_{n}$. We also denote the cylinder over the disk $\left|z_{1}\right|^{2} \leq\left(a_{1}+\varepsilon\right) / \pi$ by

$$
Z_{1, n-1}\left(a_{1}+\varepsilon\right)=\left\{\left.\left(z_{1}, \ldots, z_{n}\right)|\pi| z_{1}\right|^{2}<a_{1}+\varepsilon\right\}
$$

for $0<\varepsilon<1$.

Theorem 8.1 (Theorem 1.3). Suppose that $S>1$ and $0<\varepsilon<1$. Let $Z_{1, n-1}=Z_{1, n-1}(1+\varepsilon)$. Then we have

$$
S \leq e^{Z_{1, n-1}}(D(1, S, \ldots, S)) .
$$

Proof. We prove this inequality by contradiction. Suppose that

$$
e^{Z_{1, n-1}}(D(1, S, \cdots, S))<S
$$

and so

$$
e^{Z_{1, n-1}}(D(1, S, \ldots, S))<S-\delta
$$

for some small $\delta>0$. By definition of $e^{Z_{1, n-1}}(D(1, S, \ldots, S))$, there exists a Hamiltonian $H$ on $Z_{1, n-1}$ such that

$$
\phi_{H}^{1}(D(1, S, \ldots, S)) \cap D(1, S, \ldots, S)=\emptyset, \quad \operatorname{supp} \phi_{H} \subset Z_{1, n-1}
$$

and

$$
\|H\| \leq e^{Z_{1, n-1}}(D(1, S, \ldots, S))+\delta<S,
$$

where the inequality comes from the standing hypothesis. Since

$$
\operatorname{supp} \phi_{H} \subset \operatorname{Int} Z_{1, n-1}(1+\varepsilon)
$$

is compact, we can symplectically embed

$$
D(1, S, \ldots, S) \subset S^{2}\left(1+\varepsilon^{\prime}\right) \times \underbrace{S^{2}(\lambda) \times \cdots \times S^{2}(\lambda)}_{(n-1) \text { times }}=: X
$$

together with the image of $D(1, S, \ldots, S)$ by the isotopy $\phi_{H}^{t}, 0 \leq t \leq 1$, for some $\varepsilon^{\prime}$ with $0<\varepsilon<\varepsilon^{\prime}$ and sufficiently large $\lambda>0$. For the later purpose, we take $\varepsilon^{\prime}$ and $\lambda$ which satisfy $0<\varepsilon<\varepsilon^{\prime}<1$ and $\lambda>2 S$.

We consider a circle $S^{1}(S) \subset S^{2}(\lambda)$ which divides $S^{2}(\lambda)$ into two domains of areas $S$ and $\lambda-S$ respectively. Then we consider the torus

$$
L=L\left(\frac{1+\varepsilon^{\prime}}{2}, S, \ldots, S\right)=S^{1}\left(\frac{1+\varepsilon^{\prime}}{2}\right) \times S^{1}(S) \times \cdots \times S^{1}(S),
$$

which is a subset of $D(1, S, \ldots, S)$ because $\varepsilon^{\prime}<1$. This torus $L$ is displaceable by $\phi_{H}$ inside $X=S^{2}\left(1+\varepsilon^{\prime}\right) \times \underbrace{S^{2}(\lambda) \times \cdots \times S^{2}(\lambda)}_{(n-1) \text { times }}$ since $D(1, S, \ldots, S)$ is so. Therefore, we have $e^{X}(L) \leq\|H\|$ which follows from the definition of $e^{X}$. In particular, by (8.1) we have

$$
e^{X}(L)<S .
$$


On the other hand, we know that the torus

$$
L=L\left(\frac{1+\varepsilon^{\prime}}{2}, S, \ldots, S\right) \subset S^{2}\left(1+\varepsilon^{\prime}\right) \times \underbrace{S^{2}(\lambda) \times \cdots \times S^{2}(\lambda)}_{(n-1) \text { times }}
$$

is one of the toric fiber. By Proposition 4.3 of [4] it is weakly unobstructed (i.e., $\mathcal{M}_{\text {weak }}(L) \neq \emptyset$ ) and we can choose a weak bounding cochain $b \in$ $\mathcal{M}_{\text {weak }}(L)$ as $b=0$. Namely, $b=0$ is a weak Maurer-Cartan element without introducing bulk deformations, i.e., $\mathbf{b}=\mathbf{0} \in \mathcal{M}_{\text {weak, def }}(L)$.

Now, it remains to show

Lemma 8.2. Choose the weak bounding cochain $b=0$. Then, we have

$$
\mathfrak{T}(L) \geq S \text {. }
$$

Proof. It is enough to show that $\mathfrak{T}(L, \mathbf{0}) \geq S$ with $\mathbf{0} \in \mathcal{M}_{\text {weak, } \operatorname{def}}(L)$. By a result of $[\mathbf{2}]$ the Maslov index 2 disks are completely classified. They consist of the obvious ones coming from the upper and lower hemispheres of $S^{2}(1+$ $\left.\varepsilon^{\prime}\right)$ which have equal areas $\frac{1+\varepsilon^{\prime}}{2}$, and those two domains coming from $S^{2}(\lambda) \backslash$ $S^{1}(S)$. The coboundary map $\mathfrak{m}_{1}$ of the Floer cochain complex of $L$ are contributed by these disks. Since $\varepsilon^{\prime}<1<S$, the holomorphic disks with the minimal area are the first two disks

$$
D_{ \pm}^{2}\left(\frac{1+\varepsilon^{\prime}}{2}\right) \times\{p t\} \times \cdots \times\{p t\} \subset X
$$

which cancel each other in the operation of $\mathfrak{m}_{1}$. See Case I-a in Section 3.7.6 [3] and Theorem $1.3[6]$ for this cancellation argument. The area of the next smallest area disk is $S$ because $\lambda>2 S$. We have $(n-1)$ holomorphic disks with area $S$ :

$$
\{p t\} \times \cdots \times D_{l}^{2}(S) \times\{p t\} \times \cdots \times\{p t\} \subset X, \quad l=2, \ldots, n,
$$

where $D_{l}^{2}(S)$ is the disk with area $S$ bounding the circle $S^{1}(S)$ in the $l$ th factor $S^{2}(\lambda)$ of $X$. We note that such holomorphic disks contribute to $\mathfrak{m}_{1}$ with the same sign. (See Theorem 11.1 (3) in [4] for more general result on orientations of moduli spaces of the Maslov index 2 disks in toric manifolds.) In particular, these holomorphic disks do not cancel each other. Then the argument similar to one of Case I-b in Section 3.7.6 [3] shows that they produce a torsion part $\Lambda_{0, \text { nov }} / T^{S} \Lambda_{0, \text { nov }}$ in the Floer cohomology of $L$. It follows that $\mathfrak{T}(L, \mathbf{0}) \geq S$.

Combining (8.2) and this lemma, we obtain

$$
e^{X}(L)<\mathfrak{T}(L)
$$

However, this contradicts to Theorem 7.2 and finishes the proof of Theorem 8.1. 
By a similar argument, we can show the following variant of Theorem 8.1. We consider the domain

$$
D_{n-k, k}(1, S):=D^{2}(1)^{n-k} \times B^{2 k}(k S)
$$

for $k=1, \ldots, n-1$. Here $B^{2 k}(k S)$ is the ball in $\mathbb{C}^{k}$ of radius $\sqrt{k \pi^{-1} S}$.

Theorem 8.3 (Theorem 1.4). Suppose that $S>1$ and $0<\varepsilon<1$. Let $Z=Z_{n-k, k}(1+\varepsilon)=D^{2}(1+\varepsilon)^{n-k} \times \mathbb{C}^{k}$. Then we have

$$
S \leq e^{Z_{n-k, k}}\left(D_{n-k, k}(1, S)\right) .
$$

Proof. The proof will be the same as that of Theorem 8.1 with the following modifications. We again prove this by contradiction. Suppose that $e^{Z_{n-k, k}}\left(D_{n-k, k}(1, S)\right)<S$ and choose $\delta>0$ and $H$ as before so that

$$
e^{Z_{n-k, k}}\left(D_{n-k, k}(1, S)\right)<S-\delta
$$

and

$$
\phi_{H}^{1}\left(D_{n-k, k}(1, S)\right) \cap D_{n-k, k}(1, S)=\emptyset, \quad \operatorname{supp} \phi_{H} \subset Z_{n-k, k}(1+\varepsilon),
$$

and

$$
\|H\| \leq e^{Z_{n-k, k}}\left(D_{n-k, k}(1, S)\right)+\delta<S .
$$

Then we can symplectically embed

$$
D_{n-k, k}(1, S) \subset S^{2}\left(1+\varepsilon^{\prime}\right)^{n-k} \times \mathbb{C} P^{k}(\lambda)=: X
$$

together with the image of $D_{n-k, k}(1, S)$ by the isotopy $\phi_{H}^{t}, 0 \leq t \leq 1$, for some $\varepsilon^{\prime}$ with $0<\varepsilon<\varepsilon^{\prime}<1$ and sufficiently large $\lambda>0$. Then we consider the torus

$$
\begin{aligned}
L & =S^{1}\left(\frac{1+\varepsilon^{\prime}}{2}\right)^{n-k} \times S^{1}(S)^{k} \\
& \subset S^{2}\left(1+\varepsilon^{\prime}\right)^{n-k} \times B^{2 k}(k S) \subset S^{2}\left(1+\varepsilon^{\prime}\right)^{n-k} \times \mathbb{C} P^{k}(\lambda) .
\end{aligned}
$$

The torus $L$ is also contained in $D_{n-k, k}(1, S)$ because $\varepsilon^{\prime}<1$. Note that $L$ is one of the toric fiber in $X=S^{2}\left(1+\varepsilon^{\prime}\right)^{n-k} \times \mathbb{C} P^{k}(\lambda)$. The rest of the proof is similar to one of Theorem 8.1. So we omit it.

\section{Appendix.}

In this appendix, we give the construction of weakly filtered $A_{\infty}$-bimodule homomorphism $C\left(L^{(1)}, L^{(0)} ; \Lambda_{\text {nov }}\right) \rightarrow C\left(L^{(1) \prime}, L^{(0) \prime} ; \Lambda_{\text {nov }}\right)$ using (4.14) with $\chi=\chi_{+}$in $(2.4)$. Let $[p, w]$, resp. $\left[q^{\prime \prime}, w^{\prime \prime}\right]$, be a generator of $C\left(L^{(1)}, L^{(0)} ; \Lambda_{\text {nov }}\right)$, resp. $C\left(L^{(1) \prime}, L^{(0) \prime} ; \Lambda_{\text {nov }}\right)$. Consider the perturbed Cauchy-Riemann equation for $u: \mathbf{R} \times[0,1] \rightarrow X$

$$
\left\{\begin{array}{l}
\frac{\partial u}{\partial \tau}+J\left(\frac{\partial u}{\partial t}-\chi_{+}(\tau) X_{H}(u)\right)=0 \\
u(\tau, 0) \in L^{(0)}, u(\tau, 1) \in L^{(1)}
\end{array}\right.
$$


such that

$$
\lim _{\tau \rightarrow-\infty} u(\tau, \cdot)=\ell_{p}, \quad \lim _{\tau \rightarrow \infty} u(\tau, \cdot)=\mathfrak{g}_{H^{(0)}, H^{(1)}}^{+}\left(\ell_{q^{\prime \prime}}\right), \quad \Phi_{H^{(0)}, H^{(1)}}(w \# u) \sim w^{\prime \prime} .
$$

Let $k_{i}, i=0,1$ be non-negative integers. Consider strictly increasing sequence of real numbers $\vec{\tau}^{(i)}=\left(\tau_{1}^{(i)}, \ldots, \tau_{k_{i}}^{(i)}\right), i=0,1$. If $k_{i}=0$, we regard $\vec{\tau}^{(i)}=\emptyset$. Put marked points $\left(\tau_{j}^{(i)}, i\right)$, with $i=0,1$ and $j=1, \ldots, k_{i}$ on the boundary of the domain $\mathbf{R} \times[0,1]$.

We denote by

$$
\stackrel{\circ}{\mathcal{M}}_{k_{1}, k_{0}}\left(L^{(1)}, L^{(0)} ; J, H, \chi_{+} ;[p, w],\left[q^{\prime \prime}, w^{\prime \prime}\right] ; \operatorname{top}(\tau)\right)
$$

the moduli space of $\left(u ; \vec{\tau}^{(1)}, \vec{\tau}^{(0)}\right)$ solutions of (4.14) with boundary marked points determined by $\vec{\tau}^{(i)}, i=0,1$.

Next we recall the moduli space $\mathcal{M}_{k+1}^{\text {main }}\left(X, L^{(i)},\left\{J_{\rho}\right\}_{\rho}: \beta\right.$; top $\left.(\rho)\right)$, which is defined in [3] Definition 4.6.8. Let $J=\left\{J_{\rho}\right\}_{\rho}$ be a one-parameter family of compatible almost complex structures. (In our case, $J_{\rho}$ is independent of $\rho$.) Consider a system $\left((\Sigma, \vec{z}),\left(u_{\alpha}\right),\left(\rho_{\alpha}\right)\right)$ with the following properties:

- $\Sigma$ is a union $\cup_{\alpha} \Sigma_{\alpha}$. Here $\Sigma_{\alpha}$ are copies of the disc $D^{2}$ such that the dual graph of $\Sigma$ is a tree and $\vec{z}$ boundary marked points on $\partial \Sigma$, which do not coincide with boundary nodes. Among $\vec{z}$, there is a special marked point $z_{0}$. Denote by $\Sigma_{\alpha_{0}}$ the component containing $z_{0}$.

- $u_{\alpha}:\left(\Sigma_{\alpha}, \partial \Sigma_{\alpha}\right) \rightarrow\left(X, L^{(i)}\right)$ is $J_{\rho_{\alpha}}$-holomorphic.

- If $z \in \Sigma_{\alpha} \cap \Sigma_{\alpha^{\prime}}$, then $u_{\alpha}(z)=u_{\alpha^{\prime}}(z)$.

- $\rho_{\alpha} \in[0,1]$. In the dual graph, if the vertex corresponding to $\Sigma_{\alpha_{2}}$ is on the shortest path from the vertex corresponding to $\Sigma_{\alpha_{1}}$ and $\Sigma_{\alpha_{0}}$, the inequality $\rho_{\alpha_{1}}<\rho_{\alpha_{2}}$ holds.

- $\left((\Sigma, \vec{z}),\left(u_{\alpha}\right)\right)$ is stable in the sense of Definition 2.1.24 in $[\mathbf{3}]$.

- The homology class of $u_{\alpha}$ is $\beta(\alpha)$ and $\beta=\sum_{\alpha} \beta(\alpha)$.

- $k+1=\# \vec{z}$.

When $(k, \beta) \neq(1,0)$, we denote by $\mathcal{M}_{k+1}^{\text {main }}\left(X, L^{(i)},\left\{J_{\rho}\right\}_{\rho}: \beta ; \operatorname{top}(\rho)\right)$ the moduli space of such systems $\left((\Sigma, \vec{z}),\left(u_{\alpha}\right),\left(\rho_{\alpha}\right)\right)$. We denote by

$$
e v_{0}: \mathcal{M}_{k+1}^{\text {main }}\left(X, L^{(i)},\left\{J_{\rho}\right\}_{\rho}: \beta ; \operatorname{top}(\rho)\right) \rightarrow L
$$

the evaluation map at $z_{0}$. When $k=1, \beta=0$, we set

$$
\begin{aligned}
& \mathcal{M}_{2}^{\text {main }}\left(X, L^{(i)}, J: 0 ; \operatorname{top}(\tau)\right)=\bigcup_{\rho}\{\rho\} \\
& \quad \times\left\{\left(u, z_{0}=1, z_{1}=-1\right) \mid u:\left(D^{2}, \partial D^{2}\right) \rightarrow\left(X, L^{(i)}\right) \text { a constant map }\right\} .
\end{aligned}
$$


We define the moduli space $\mathcal{M}_{k_{1}, k_{0}}^{\mathrm{unbr}}\left(L^{(1)}, L^{(0)} ; J, H, \rho_{+} \quad: \quad[p, w],\left[q^{\prime \prime}, w^{\prime \prime}\right]\right.$; $\operatorname{top}(\tau))$ of systems

$$
\begin{aligned}
\mathfrak{D}=\left(\left(\Sigma ; u ; \vec{\tau}^{(1)}, \vec{\tau}^{(0)}\right) ; \quad\right. & \left(\left(\Sigma_{(1), j}, \vec{z}_{(1), j}\right),\left(u_{(1), j, \alpha}\right),\left(\rho_{(1), j, \alpha}\right)\right) ; j=1, \ldots, b: \\
& \left.\left(\left(\Sigma_{(0), j}, \vec{z}_{(0), j}\right),\left(u_{(0), j, \alpha}\right),\left(\rho_{(0), j, \alpha}\right)\right) ; j=1, \ldots, a\right)
\end{aligned}
$$

with the following properties.

- $\ell_{j}^{(i)}$ are non-negative integers and $\sum_{j} \ell_{j}^{(i)}=k_{i}$.

- $\left(\Sigma ; u ; \vec{\tau}^{(1)}, \vec{\tau}^{(0)}\right) \in \stackrel{\circ}{\mathcal{M}}_{b, a}\left(L^{(1)}, L^{(0)} ; J, H, \rho_{+} ;[p, w],\left[q^{\prime \prime}, \tilde{w}\right]\right)$.

- $\left(\left(\Sigma_{(i), j}, \vec{z}_{(i), j}\right),\left(u_{(i), j, \alpha}\right),\left(\rho_{(i), j, \alpha}\right)\right) \in \mathcal{M}_{\ell_{j}^{(i)}+1}^{\operatorname{main}}\left(X, L^{(i)}, J ; \beta_{j}^{(i)}: \operatorname{top}(\rho)\right)$, $i=0,1$. Here we take $J=J_{\rho}$ is independent of $\rho$.

- $e v_{0}\left(\left(\Sigma_{(i), j}, \vec{z}_{(i), j}\right),\left(u_{(i), j, \alpha}\right),\left(\rho_{(i), j, \alpha}\right)\right)=u\left(\tau_{j}^{(i)}, i\right)$.

- $\rho_{(i), j, \alpha_{0}} \leq \chi\left(\tau_{j}^{(i)}\right)$.

- $\Phi_{H^{(0)}, H^{(1)}}\left(\sum_{i=0}^{1} \sum_{j} \beta_{j}^{(i)} \# w \# u\right) \sim w^{\prime \prime}$.

Here we note that some of $\ell_{j}^{(i)}=1$ and $\beta_{j}^{(i)}=0$. Then $\Sigma_{(i), j}$ is irreducible and $u_{(i), j}$ is a constant map. We may identify it with a boundary marked point on $\mathbf{R} \times[0,1]$.

Finally, we define the moduli space

$$
\begin{aligned}
& \mathcal{M}_{k_{1}, k_{0}}\left(L^{(1)}, L^{(0)} ; J, H ;[p, w],\left[q^{\prime \prime}, w^{\prime \prime}\right]: \operatorname{top}(\tau)\right) \\
= & \coprod \mathcal{M}_{\ell_{1}, \ell_{0}}\left(L^{(1)}, L^{(0)} ; J ;[p, w],\left[p^{\prime}, w^{\prime}\right]\right) \\
& \times \mathcal{M}_{k_{1}-\ell_{1}-m_{1}, k_{0}-\ell_{0}-m_{0}}^{\text {unbr }}\left(L^{(1)}, L^{(0)} ; J, H, \chi_{+} ;\left[p^{\prime}, w^{\prime}\right],\left[q, \tilde{w}^{\prime}\right] ; \operatorname{top}(\tau)\right) \\
& \times \mathcal{M}_{m_{1}, m_{0}}\left(L^{(1) \prime}, L^{(0) \prime} ; J ;\left[q, \tilde{w}^{\prime}\right],\left[q^{\prime \prime}, w^{\prime \prime}\right]\right) .
\end{aligned}
$$

Here we identify $\mathcal{M}_{m_{1}, m_{0}}\left(L^{(1) \prime}, L^{(0) \prime} ; J ;\left[q, \tilde{w}^{\prime}\right],\left[q^{\prime \prime}, w^{\prime \prime}\right]\right)$ with the moduli space of solutions of the perturbed Cauchy-Riemann equation (4.14) as we explained before stating Lemma 4.2. Then we define $\phi_{k_{1}, k_{0}}\left(P_{k_{1}}^{(1)} \otimes \cdots \otimes P_{1}^{(1)} \otimes\right.$ $\left.[p, w] \otimes P_{1}^{(0)} \otimes \cdots \otimes P_{k_{0}}^{(0)}\right)$ to be

$$
\begin{aligned}
& \sum \#\left(\mathcal{M}_{k_{1}, k_{0}}\left(L^{(1)}, L^{(0)} ; J, H:[p, w],\left[q^{\prime \prime}, w^{\prime \prime}\right] ; \operatorname{top}(\tau)\right)\right. \\
& \left.\quad \times{ }_{\left(L^{(1)}\right)^{k_{1}}}\left(P_{k_{1}}^{(1)} \times \cdots \times P_{1}^{(1)}\right) \times{ }_{\left(L^{(0)}\right)^{k_{0}}}\left(P_{1}^{(0)} \times \cdots \times P_{k_{0}}^{(0)}\right)\right)\left[q^{\prime \prime}, w^{\prime \prime}\right],
\end{aligned}
$$

where the summation is taken over all possible zero-dimensional fiber product.

Then we can prove that $\phi=\left\{\phi_{k_{1}, k_{0}}\right\}$ is a weakly filtered $A_{\infty}$-bimodule homomorphism exactly in the same way as in Sections 5.3.1 and 5.3.2 in [3]. The hamiltonian diffeomorphisms $\phi_{H^{(0)}}^{1}$, resp. $\phi_{H^{(1)}}^{1} \operatorname{map} L^{(0)}$ to 
$L^{(0) \prime}$, resp. $L^{(1)}$ to $L^{(1) \prime}$. Hence, we have isomorphisms $C\left(L^{(0)} ; \Lambda_{0, \text { nov }}\right) \rightarrow$ $C\left(L^{(0) \prime} ; \Lambda_{0, \text { nov }}\right)$, resp. $C\left(L^{(1)} ; \Lambda_{0, \text { nov }}\right) \rightarrow C\left(L^{(1) \prime} ; \Lambda_{0, \text { nov }}\right)$, of $\Lambda_{0, \text { nov }}$-modules. We can take suitable compatible almost complex structures, abstract perturbations in a compatible way so that they induce isomorphisms $\left(\phi_{H^{(0)}}^{1}\right)_{*}$ and $\left(\phi_{H^{(1)}}^{1}\right)_{*}$ of filtered $A_{\infty}$-algebras. See Chapter 3 in $[\mathbf{3}]$. Then $\left\{\phi_{k_{1}, k_{0}}\right\}$ is a filtered $A_{\infty}$-bimodule homomorphism over $\left(\phi_{H^{(1)}}^{1}\right)_{*}$ and $\left(\phi_{H^{(0)}}^{1}\right)_{*}$.

For bounding cochains $b_{i}$ of $L^{(i)}, i=0,1$, we set

$$
\Phi^{b_{1}, b_{0}}(\bullet)=\sum_{k_{1}, k_{0}} \phi_{k_{1}, k_{0}}\left(b_{1}^{\otimes k_{1}} \otimes \bullet \otimes b_{0}^{\otimes k_{0}}\right) .
$$

Then, we obtain a chain homomorphism between Floer complexes $\Phi^{b_{1}, b_{0}}$ : $C\left(L^{(1)}, L^{(0)} ; \Lambda_{\text {nov }}\right) \rightarrow C\left(L^{(1) \prime}, L^{(0) \prime} ; \Lambda_{\text {nov }}\right)$. We can extend this construction with bulk deformation as usual to obtain a chain homomorphism $\Phi^{\mathbf{b}_{\mathbf{1}}, \mathbf{b}_{\mathbf{0}}}$ for $\left(\mathbf{b}_{1}, \mathbf{b}_{0}\right) \in \mathcal{M}_{\text {weak,def }}\left(L^{(1)}\right) \times_{\left(\pi_{\text {amb }}, \mathfrak{P O}\right)} \mathcal{M}_{\text {weak,def }}\left(L^{(0)}\right)$.

\section{References}

[1] Y.V. Chekanov, Lagrangian intersections, symplectic energy, and areas of holomorphic curves, Duke Math. J. 95 (1998), no. 1, 213-226.

[2] C.-H. Cho and Y.-G. Oh, Floer cohomology and disc instantons of Lagrangian torus fibers in Fano toric manifolds, Asian J. Math. 10 (2006), 773-814.

[3] K. Fukaya, Y.-G. Oh, H. Ohta and K. Ono, Lagrangian intersection Floer theoryanomaly and obstruction, Part I, and Part II, AMS/IP Studies in Advaneced Math. 46.1, and 46.2, International Press/Amer. Math. Soc. (2009). MR2553465, MR2548482.

[4] K. Fukaya, Y.-G. Oh, H. Ohta and K. Ono, Lagrangian Floer theory on compact toric manifolds I, Duke Math. J. 151 (2010), 23-174.

[5] K. Fukaya, Y.-G. Oh, H. Ohta and K. Ono, Lagrangian Floer theory on compact toric manifolds II : bulk deformations, Selecta Mathematica, New Series, 17(3) (2011), 609-711.

[6] K. Fukaya, Y.-G. Oh, H. Ohta and K. Ono, Anti-symplectic involution and Floer cohomology, submitted, arXiv:0912.2646.

[7] K. Fukaya, Y.-G. Oh, H. Ohta and K. Ono, Toric degeneration and non-displaceable Lagrangian tori in $S^{2} \times S^{2}$, Int. Math. Res. Not. IMRN, 2012(13) (2012), 2942-2993. doi:10.1093/imrn/rnr128 arXiv: 1002.1660.

[8] R. Hind and E. Kerman, New obstructions to symplectic embeddings, preprint 2009, arXiv:0906.4296.

[9] H. Hofer, On the topological properties of symplectic maps, Proc. R. Soc. Edinburgh 115 (1990), 25-38.

[10] Y.-G. Oh, Floer cohomology of Lagrangian intersections and pseudo-holomorphic disks. I, Comm. Pure Appl. Math. 46 (1993), 949-993; Addendum, Comm. Pure Appl. Math. 48 (1995), 1299-1302,

[11] Y.-G. Oh, Symplectic topology as the geometry of action functional, I, J. Differ. Geom. 46 (1997), 499-577. 
[12] Y.-G. Oh, Gromov-Floer theory and disjunction energy of compact Lagrangian embeddings, Math. Res. Lett. 4 (1997), 895-905.

[13] A. Weinstein, Connections of Berry and Hannay type for moving Lagrangian submanifolds, Adv. Math. 82(2) (1990), 133-159.

Department of Mathematics

KYOTO UNIVERSITY

КҮОТО

JAPAN

E-mail address: fukaya@math.kyoto-u.ac.jp

Department of Mathematics

UNIVERSITY OF WISCONSIN

MADISON, WI

USA

AND

IBS Center for Geometry and Physics and POSTeCH, Pohang

Korea

E-mail address: oh@math.wisc.edu

Graduate School of Mathematics

NAGOYA UNIVERSITY

NAGOYA

JAPAN

AND

Korea Institute for Advanced Study

SEOUL

KOREA

E-mail address: ohta@math.nagoya-u.ac.jp

Research Institute for Mathematical Sciences

KYOTO UNIVERSITY

КҮОТО

JAPAN

AND

Korea Institute for Advanced Study

SEOUL

KoreA

E-mail address: ono@kurims.kyoto-u.ac.jp

Received 06/29/2011, accepted 11/09/2011

K.F. was supported partially by JSPS Grant-in-Aid for Scientific Research Nos 19104001, 23224002 and Global COE program G08, Y.O. by US NSF grant No. 0904197 and Research Center Program of IBS in Korea. H.O. by JSPS Grant-in-Aid for Scientific Research Nos. 19340017 and 23340015, and K.O. by JSPS Grant-in-Aid for Scientific Research, Nos 21244002 and 18340014 